\title{
Optimization of Transmitter-Receiver Pairing of Spaceborne Cluster Flight Netted Radar for Area Coverage and Target Detection
}

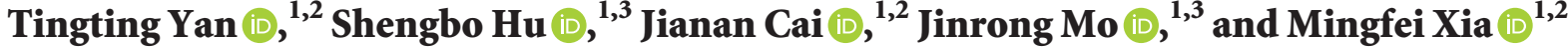 \\ ${ }^{1}$ Institute of Intelligent Information Processing, Guizhou Normal University, Guiyang 550001, China \\ ${ }^{2}$ School of Mathematical Sciences, Guizhou Normal University, Guiyang 550001, China \\ ${ }^{3}$ Center for RFID and WSN Engineering, Department of Education Guizhou, Guiyang 550001, China
}

Correspondence should be addressed to Tingting Yan; 1592624854@qq.com and Shengbo Hu; hsb@nssc.ac.cn

Received 2 September 2020; Revised 17 February 2021; Accepted 23 February 2021; Published 10 March 2021

Academic Editor: Mohamed El Ghami

Copyright (C) 2021 Tingting Yan et al. This is an open access article distributed under the Creative Commons Attribution License, which permits unrestricted use, distribution, and reproduction in any medium, provided the original work is properly cited.

In this paper, we investigate the optimization problem of the transmitter-receiver pairing of spaceborne cluster flight netted radar (SCFNR) for area coverage and target detection. First of all, we propose the novel concept of SCFNR integrated cluster flight spacecraft with netted radar, the mobility model for bistatic radar pair with twin-satellite mode, and formulate the radar-target distance distribution function and radar-target distance product distribution function with geometric probability method. Secondly, by dividing surveillance region into grids, we define the 0-1 grid coverage matrix for bistatic radar and the transmitterreceiver pairing matrix for SCFNR with using radar equation and the radar-target distance distribution function, and we describe the optimal problem of transmitter-receiver pairing of SCFNR for area coverage and target detection by defining K-grid coverage matrix. Thirdly, we propose a new algorithm integrated particle swarm optimization with Hungarian algorithm (PSO-HA) to address the optimal problem, which is actually one-to-one pairing problem. Finally, we validate the effectiveness and reasonability of the proposed algorithm through numerical analysis.

\section{Introduction}

As a distributed space system, the spaceborne netted radar is composed of several spatially separated, mutually independent, and cooperative radars in space. Compared with the traditional radar, spaceborne netted radar has advantages of high flexibility, reliability, and antistealth ability [1-3]. In addition, it also has the advantage of being allweather, wide coverage, and satisfying specific coverage requirements due to its location in outer space $[4,5]$. On the other hand, the cluster flight spacecraft has been one of the hot issues regarding the distributed space network, because of its advantages of flexibility, rapid response, low cost, strong scalability, and long lifetime [6-8]. Unlike traditional satellite formation flying applications, cluster flight spacecraft requires nodes to maintain bounded relative distances between tens or hundreds of kilometers and to keep loose geometry for the entire mission lifetime, so that orbit controlling and relative position sensing for the spacecraft can be performed well [6-8]. Some researches are mentioned cluster flight spacecraft. The paper [6] presents clusterkeeping algorithms aimed at minimizing fuel consumption. The paper [9] provides a cooperative control framework aimed at synchronizing the mean-orbital element convergence among cluster-flying satellites. The paper [10] studies the relationship between first docking time and spatial initial distribution and the relationship between first separating time and spatial initial distribution for cluster flight spacecraft. The influence of node transmit power on the QoS performance of cluster flight spacecraft network is analyzed in [11]. In order to improve the performance of cluster flight spacecraft network, the nodal distance distributions are studied in [12]. Hence, we propose the novel concept of SCFNR integrated cluster flight spacecraft with the 
spaceborne netted radar, and the optimization problem of SCFNR on coverage is addressed.

Coverage is one of the important issues about radar for target detection, localization, and tracking. According to radar equations, the coverage problem of the spaceborne netted radar is related to many factors such as orbit, antenna gain, transmitted power, and radar cross section. To meet the need of improving the target detection and position, it expects that more radars of SCFNR can cover the surveillance region on the earth, which is completely different from line-of-sight (LOS) coverage of satellite constellation $[13,14]$. When the antenna gain, the transmitted power, and radar cross section are constant, the coverage of SCFNR completely depends on the product of transmitter-target and target-receiver distance and the spacecraft orbit. Our previous research shows that the geometry configuration of SCFNR is characterized by high spatiotemporal dynamic and random, which complicates coverage problem of SCFNR. So, the problem about the coverage about SCFNR is more challenging.

The netted radar is a case of multistatic radar [15-19], where transmitters can collaborate with several receivers at different locations. According to the pairing method of transmitter-receiver, the netted radar is mainly classified into three categories: a group of bistatic radars, a single transmitter with several receivers, and a single receiver with several transmitters. To improve the performance of SCFNR, it expects that more radars of SCFNR can cover the surveillance region, and this can be described by the maximum intersection coverage. Actually, the maximum intersection coverage is the classical maximum $k$-subset intersection (MSI) in graph theory, and it is also a combinatorial optimization problem [20]. To the best of our knowledge, there is not seen much on solving MSI problems. In [21], the authors introduce a GRASP heuristic and propose an integer programming formulation MSI problem. However, to solve the MSI problem about SCFNR is more difficult due to the geometry configuration with spatiotemporal dynamic and random.

To the best of our knowledge, this makes the first paper to investigate the SCFNR coverage problem. The main contributions of our work are summarized as follows:

(1) We propose the novel concept of SCFNR integrated cluster flight spacecraft with netted radar, and the mobility model for bistatic radar pair is established by twinsatellite mode. The distribution function of the product of transmitter-target and receiver-target distance is derived using the method of geometric probability.

(2) According to radar equation, we propose the concept of $0-1$ grid coverage matrix for bistatic radar by dividing the surveillance region into grids, and the definition of the transmitter-receiver pairing matrix for SCFNR is given using bistatic radar pairs. These provide an important theoretical basis for optimizing the transmitter-receiver pairing of SCFNR for area coverage and target detection.

(3) We describe the optimal problem of transmitter-receiver pairing of SCFNR for area coverage and target detection by defining $K$-grid coverage matrix. Also, we propose a new algorithm integrated PSO-HA to address the optimal problem. We validate the effectiveness of the proposed algorithms through numerical calculation.

The rest of the paper is organized as follows: Section 2 reviews the related work. Section 3 proposes the novel concept of SCFNR, establishes mobility model for bistatic radar pair, and derives the distribution function of the product of transmitter-target and receiver-target distance. Section 4 defines the coverage matrix of bistatic radar and pairing matrix of SCFNR based on Section 3 and describes the optimal problem of transmitter-receiver pairing of SCFNR for area coverage and target detection. Section 5 presents the PSO-HA algorithm. Section 6 verifies the effectiveness of the proposed algorithm, and coverage and detection results using numerical calculation are given. Finally, we conclude the paper in Section 7.

\section{Related Works}

In recent years, with continuing advances in communication technology and micro-electromechanical systems (MEMS) technology, multistatic radar sensing technology has received considerable attention, especially bistatic radar sensing coverage. For instance, in [22], the authors considered the problem of deploying a network of bistatic radars in a region to maximize the worst-case intrusion detectability. They studied the coverage problem of a bistatic radar sensor network and the optimal placement of bistatic radars on a line segment to minimize its vulnerability. In [23], Wang et al. studied the belt barrier coverage with the minimum total placement cost in bistatic radar sensor networks. They proposed a line-based equipartition placement strategy such that all radars placed on a deployment line can form a barrier with some breadth and one or more such placement lines can form a belt barrier with the required breadth. In [24], the authors studied area coverage in bistatic radar sensor networks. They investigated the geometrical relationship between the $c$-coverage area of a bistatic radar and the distance between its component transmitter and receiver. Then, they reduced the problem dimension by transforming the area coverage problem to point coverage problem by employing the intersection point concept. In [25], the authors studied the worst-case coverage under deterministic deployment, aiming to find optimal deployment locations of radar transmitters and receivers such that the worst-case intrusion detectability was maximized. Then, by developing a novel 2-site Voronoi diagram with graph search techniques, they designed an algorithm to find approximate worst-case intrusion detectability. In [23], the authors studied the belt barrier coverage in bistatic radar sensor networks, which was dependent on the distance between a pair of radar transmitter and receiver. In [26], Wang et al. studied barrier coverage in bistatic radar sensor networks. They formulated the barrier coverage problem as minimum weight barrier coverage problem. By constructing a directed coverage graph, minimum weight barrier coverage problem was transformed into finding $k$ node-disjoint shortest paths. Next, they proposed an energy-efficient algorithm to solve the problem within polynomial. 
In addition, intelligent coverage becomes a practical research topic in dynamic sensors network. The paper [27] provided wireless signal coverage schemes for point-to-point and pointto-region and determined the required horizontal rotation angle and pitch rotation angle of the directional antenna intelligent coverage. In [28], the authors established the mobile sensor noncooperative game model. Then, a local information-based topology control (LITC) algorithm based on this model was proposed, in which sensors move to enhance coverage by exchanging information with neighbors. Also, the application of PSO is wide in complex system. In [29], a systematic data-driven adaptive neuro-fuzzy inference system (ANFIS) modelling methodology was proposed, and a high-performance PSO-LSE method was developed to improve the structure and to identify the consequent parameters of ANFIS model. In [30], the authors proposed an algorithm combined with belief-desire-intention agent with a quantum-behaved particle swami optimization (QPSO) algorithm to optimize a marine generator excitation controller, and the QPSO algorithm was highly robust because its performance was insensitive to the accuracy of system parameters. For intelligent coverage in complex dynamic environment, in [31], a novel trajectory scheduling method based on coverage rate for multiple mobile sinks was presented, especially for large-scale wireless sensor networks, and an improved PSO combined with mutation operator was introduced to search the parking positions with optimal coverage rate. Predictably, considering complex dynamic sensor network, PSO in intelligent coverage is helpful.

For a long time, some works on spaceborne radar coverage are mainly focused on optimizing the orbital design according to the observation and detection requirements. For example, in [32], the authors presented a feasibility analysis of a spaceborne bistatic radar mission for soil moisture retrieval, and they studied the assessment of the spatial coverage from orbital design. In [33], the authors adopted bistatic geometry from space platforms, and they implemented bistatic synthetic aperture radar observation. In [34], based on the analysis of radar cross-section (RCS) characteristic of geostationary orbital targets, the orbital altitude and revisiting period of space-based radar was designed in detail, and they discussed the relationship between image's resolutions of spaceborne inverse synthetic aperture radar and system parameters. In [35], the authors established a spaceborne-airborne bistatic radar model, and then they analyzed moving target detecting performance of the space-time adaptive processing technology.

For cluster flight spacecraft, related researches focus more on orbital control and node connection. The paper [6] presented a methodological development of cluster flight algorithms for disaggregated satellite systems in low Earth orbits. To obtain distance-bounded relative motion, a new constraint on the initial conditions of the modules was developed. In [9], the authors developed the implementable cluster flight-control methods with realistic orbital and actuator modelling. They offered two distributed orbit control laws with fixed-magnitude thrust for satellite cluster flight based on mean-orbital elements. Recently, the team of this paper has done some works on the cluster flight spacecraft network. For example, in [10], the authors proposed the constraint condition of orbital elements for noise-limited fractionated spacecraft network percolating and path formation time. The numerical results showed that the network topology for fractionated spacecraft is time varying and dynamic. The paper [11] investigated the transmit power allocation problem to minimize the average packet error rate at the access point in the cluster flight spacecraft network. Due to the complexity of the calculation, the probability density function of the distance between nodes was fitted using eighthorder polynomial.

\section{The Concept of SCFNR}

As mentioned above, the spaceborne netted radar is composed of several spatially separated, mutual independent, and cooperative radars in space. The spaceborne netted radar has the advantages of high flexibility, reliability, and antistealth ability. In addition, it also has the advantage of being all-weather, wide coverage, and satisfying specific coverage. On the other hand, the cluster flight spacecraft has many advantages such as flexibility, rapid response, low cost, strong scalability, and long lifetime. What's more, cluster flight spacecraft can perform orbit controlling and relative position sensing easily. Hence, we propose the novel concept of SCFNR integrated advantages of both cluster flight spacecraft and the spaceborne netted radar.

Generally, netted radar has the following three cases: (1) a group of bistatic radars, where the output of the bistatic radars are processed centrally to obtain a decision regarding the presence of a target and to estimate parameters. In this case, it is assumed that the transmitters do not interfere with each other, which is typically achieved either by using separate frequency bands or orthogonal transmitted waveforms. At the same time, each receiver is assumed to be able to receive the signals from each transmitter; (2) a single transmitter with several receivers, typically in the case of a high-value unit equipped with the transmitter, for instance an airborne warning and control system, and receivers cooperating to achieve the detection; (3) a single receiver with several transmitters, where a single receiver receives waveforms from several transmitters in different frequency bands to information fusion.

In this paper, we adopt SCFNR with bistatic radar pairs. It is assumed that one-to-one pairing method is taken by SCFNR in any slot of the orbital hyperperiod. So, we assume that each pair of transmitter and receiver can potentially form a bistatic radar. We further assume that orthogonal transmissions are used for interference avoidance. In view of this, we assume that one transmitter can only be connected to one receiver, and the corresponding bistatic radar is formed in any slot of the orbital hyperperiod. Therefore, given a SCFNR consisting of $N$ radars, if $N$ is even, then the pairing of bistatic radars is $N / 2$ pairs, and if $N$ is odd, then the pairing of bistatic radars is $(N-1) / 2$ pairs and a monostatic radar. Since the monostatic radar can be considered as a bistatic radar with a baseline length 0 , it can also be considered that $(N+1) / 2$ pairs of bistatic radars is formed.

Based on the above, this paper focuses on the optimization problem of the transmitter-receiver pairing of SCFNR for area of interest coverage and target detection, that is, how to pair transmitter-receiver properly to satisfy the requirements of area coverage and detection in any slot of the orbital hyperperiod. First, the mobility model for SCFNR is 
presented and analyzed. Of course, the mobility model for bistatic radar pair can be given, and also the distribution function of the product of transmitter-target and receivertarget distance needs to be derived.

3.1. The Mobility Model for Bistatic Radar Pair. To accomplish the cluster flight model within bounded distance, the twin-satellite model is adopted to study the mobility model for bistatic radar pair. As shown in Figure 1, the transmitter or receiver position is uniformly distributed on sphere within $(M-m) / 4 . M$ is the upper bound of transmitterreceiver distance in SCFNR, and $m$ is the lower bound.

Based on orbit dynamics theory, the orbital hyperperiod can be divided into $\mathscr{T}_{0}, \mathscr{T}_{1}, \mathscr{T}_{2}, \ldots, \mathscr{T}_{\mathscr{T}}$, times for fractionated spacecraft $[7,36]$. So, there are $\mathscr{T}$ time slots in an orbital period. The orbital hyperperiod is $\mathscr{H}=\left(\mathscr{T}_{\mathscr{T}}-\mathscr{T}_{0}\right)$, time slot $\sigma_{k}=\left[\mathscr{T}_{k-1}, \mathscr{T}_{k}\right)(k=1,2, \ldots, \mathscr{T})$ [7]. So, the mobility model of SCFNR can be defined as follows.

Definition 1. In earth-centered inertial (ECI) coordinates, if the position set of $N$ transmitters and receivers in CFSNR is $S(0)=$ $\left\{S_{1}(0), S_{2}(0), \ldots, S_{N}(0)\right\}$ at initial time $\mathscr{T}_{0}$, the position set is $\mathbf{S}(k)=\left\{S_{1}(k), S_{2}(k), \ldots, S_{N}(k)\right\}$, and the positions are uniformly distributed within sphere $B\left(S_{i}(0), R\right)(i=1,2, \ldots, N)$ at time $k$, where $S_{i}(0)$ and $R=(M-m) / 4$ are the center and radius of the sphere, respectively. Moreover, positions among all transmitters and receivers are mutually independent and independent of all previous locations.

3.2. The Probability Distribution Function of the Distance Product. We consider a SCFNR scenario as shown in Figure 2. Let $\mathbf{T}$ be the transmitter set and $\mathbf{R}$ be the receiver set. Transmitters and receivers are located at different locations. We use TR to denote all transmitter-receiver pairs. If transmitter $T_{i} \in \mathbf{T}$ and receiver $R_{j} \in \mathbf{R}$ choose the same channel, then the bistatic radar $T_{i} R_{j} \in$ TR is formed by $T_{i}$ and $R_{j}$, and different channels can be considered as orthorhombic channels to avoid interference. Without ambiguity, in any time slot of orbital hyperperiod for SCFNR, the position of transmitter and receiver is denoted by $S_{i T}$ and $S_{j R}$, respectively, where $i \neq j$.

Thus, in SCFNR scenario, let $\mathrm{P}$ be a target position in the surveillance region. According to [37], for a bistatic radar $T_{i} R_{j} \in \mathrm{TR}$, the signal-to-noise ratio (SNR) of $\mathrm{P}$ can be given as

$$
\mathrm{SNR}=\frac{K_{B}}{\left\|S_{i T} P\right\|^{2}\left\|P S_{j R}\right\|^{2}},
$$

where $\left\|S_{i T} P\right\|$ and $\left\|P S_{j R}\right\|$ denote transmitter-target and target-receiver distances, respectively. $K_{B}$ is a constant related to the physical-layer parameters of the bistatic radar, such as transmit power, antenna gains of transmitter and receiver, and radar cross-section. However, we are not interested in the abovementioned physical-layer parameters, but transmitter-target and target-receiver distances. For convenience, we assume that the constant is identical for any bistatic radar, i.e., homogeneous bistatic radar also.

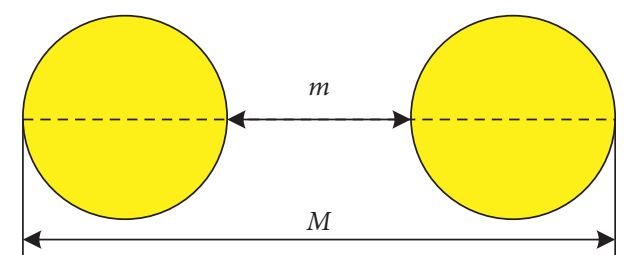

FIgURE 1: The mobility model for bistatic radar pair.

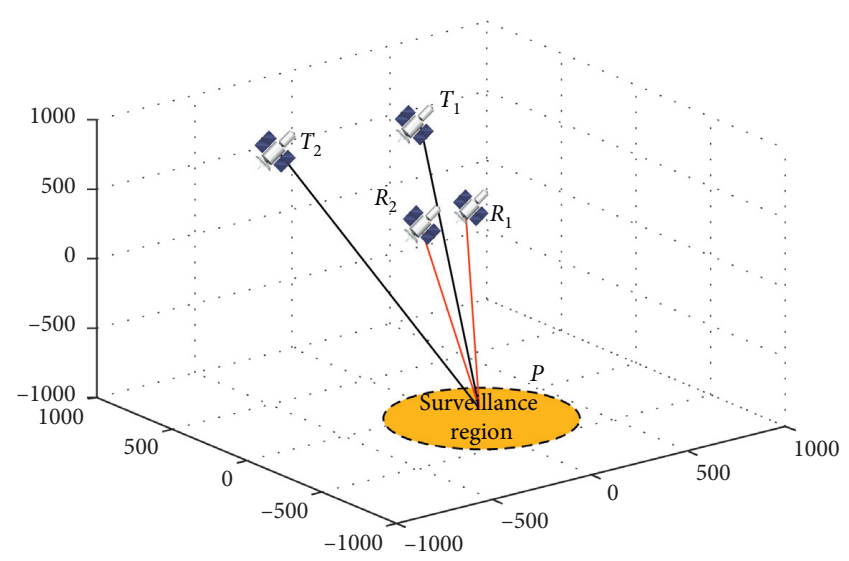

Figure 2: The SCFNR scenario.

As seen from equation (1), the SCFNR performance is determined by $\left\|S_{i T} P\right\|\left\|P S_{j R}\right\|$, i.e., the product of transmittertarget and target-receiver distances. According to Definition 1 , the product is random. Therefore, we need to analyze its distribution.

For convenience, the 2D scenario about transmitter $T_{i}$ and target $P$ in SCFNR is described in Figure 3. $T_{i}$ is assumed to be uniformly located in a circle of the twodimensional plane, and the $P$ is assumed to be fixed. In Figure 3, let $\left\|S_{i T} P\right\|=d_{i}, h_{i}$ be the distance between $P$ and initial orbital position of $T_{i}$, where $d_{i} \in\left[h_{i}-R, h_{i}+R\right]\left(h_{i}>R\right)$. Actually, if $P$ is a target position of earth surface, then $h_{i}$ can be considered as the orbit height of $T_{i}$ at initial time.

Therefore, the transmitter-target distance $d_{i}$ has the distance function given by probability distribution, that is,

$$
F_{D_{i}}\left(d_{i}\right) \triangleq P\left\{D_{i} \leq d_{i}\right\}, \quad h_{i}-R \leq d_{i} \leq h_{i}+R, h_{i}>R .
$$

Here, $F_{D_{i}}\left(d_{i}\right)$ is calculated with geometric probability method [38, 39].

Now, we extend the 2D scenario in Figure 3 into 3D scenario. Let $\Omega$ be the sphere $O$ and $C_{0}$ be the intersection volume between sphere $O$ and the sphere of radius $d_{i}$ centered at $P$. Equation (2) can be rewritten as

$$
F_{D_{i}}\left(d_{i}\right)=\frac{\mu\left(C_{0}\right)}{\mu(\Omega)},
$$

where $\mu(\Omega)=4 \pi R^{3} / 3$ is the measure of $\Omega$.

In order to calculate $F_{D_{i}}\left(d_{i}\right), \mu\left(C_{0}\right)$ can be divided into two cases: (1) $d_{i} \in\left[h_{i}-R, h_{i}\right) ;(2) d_{i} \in d_{i} \in\left[h_{i}, h_{i}+R\right]$. Thus, Theorem 1 about distribution function of $d_{i}$ can be proved. 


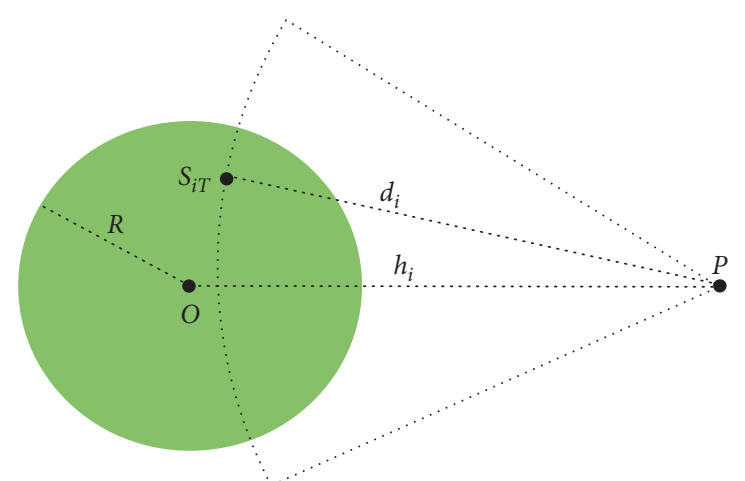

FIGURE 3: The transmitter-target distance distribution in SCFNR.

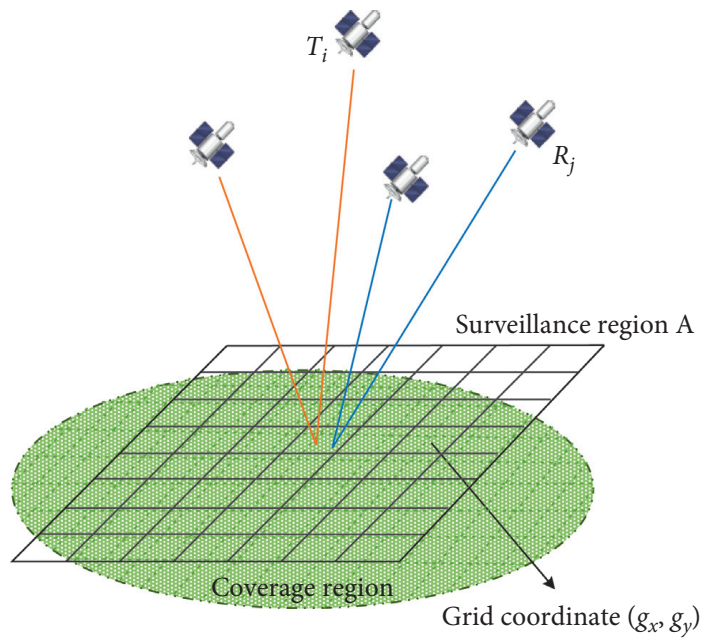

FIGURE 4: Sketch of coverage region in SCFNR scenario.

Theorem 1. In SCFNR, if the initial position of transmitter $S_{i T}(0)$ and mobility model $M(t)$ are given, then the distribution function of random variable $d_{i}$ is

$$
F_{D_{i}}\left(d_{i}\right)= \begin{cases}\frac{0,}{2 d_{i}^{3}+h_{a}^{3}-3 h_{a} d_{i}^{2}+2 R^{3}+h_{b}^{3}-3 h_{b} R^{2}} & d_{i}<h_{i}-R, \\ 4 R^{3} & h_{i}-R \leq d_{i}<h_{i}, \\ \frac{2 d_{i}^{3}+h_{a}^{3}-3 h_{a} d_{i}^{2}+2 R^{3}-h_{c}^{3}+3 h_{c} R^{2}}{4 R^{3}}, & h_{i} \leq d_{i} \leq h_{i}+R, \\ 1, & h_{i}+R<d_{i},\end{cases}
$$

where $d_{i}$ is the radar-target distance, $h_{a}=\left(h_{i}^{2}-R^{2}+d_{i}^{2} / 2 h_{i}\right)$, $h_{b}=\left(h_{i}^{2}+R^{2}-d_{i}^{2} / 2 h_{i}\right)$, and $h_{c}=\left(-h_{i}^{2}-R^{2}+d_{i}^{2} / 2 h_{i}\right)$.

Proof of Theorem 1. The proof of Theorem 1 is given in Appendix A.
Similarly, if $\left\|P S_{j R}\right\|=d_{j}$ is the distance between target $P$ and receiver $R_{j}$, then $\left\|S_{i T} P\right\|\left\|P S_{j R}\right\|=d_{i} d_{j}=d_{i j}$ is the product of transmitter-target and target-receiver distances. Since $d_{i}$ and $d_{j}$ are independent, Theorem 2 about distribution function of $d_{i j}$ can be proved.

Theorem 2. In SCFNR, if the initial positions of transmitter $S_{i T}(0)$, receiver $S_{j R}(0)$, and mobility model $M(t)$ are given, then distribution function of random variable $d_{i j}$ is

$$
F_{D_{i j}}\left(d_{i j}\right)= \begin{cases}0, & d_{i j}<\left(h_{1}-R\right)\left(h_{2}-R\right), \\ F_{D_{i j}}^{1}\left(d_{i j}\right), & \left(h_{1}-R\right)\left(h_{2}-R\right) \leq d_{i j}<\left(h_{1}+R\right)\left(h_{2}-R\right), \\ F_{D_{i j}}^{2}\left(d_{i j}\right), & \left(h_{1}+R\right)\left(h_{2}-R\right) \leq d_{i j}<\left(h_{1}-R\right)\left(h_{2}+R\right), \\ F_{D_{i j}}^{3}\left(d_{i j}\right), & \left(h_{1}-R\right)\left(h_{2}+R\right) \leq d_{i j} \leq\left(h_{1}+R\right)\left(h_{2}+R\right), \\ 1, & \text { otherwise, }\end{cases}
$$

where $h_{1}=\max \left\{h_{i}, h_{j}\right\}, h_{2}=\min \left\{h_{i}, h_{j}\right\}$, and $h_{j}$ is the orbit height of radar receiver $R_{j}$ at initial time, 


$$
\begin{aligned}
& F_{D_{i j}}^{1}\left(d_{i j}\right)=\frac{C_{1}^{1} d_{i j}^{2}}{2}+\frac{C_{2}^{1} d_{i j}^{3}}{3}+\frac{C_{3}^{1} d_{i j}^{4}}{4}+\frac{C_{4}^{1} d_{i j}^{2}}{4}\left(2 \ln \frac{d_{i j}}{\left(h_{1}-R\right)\left(h_{2}-R\right)}-1\right)+\frac{C_{5}^{1} d_{i j}^{3}}{9}\left(3 \ln \frac{d_{i j}}{\left(h_{1}-R\right)\left(h_{2}-R\right)}-1\right) \frac{C_{6}^{1} d_{i j}^{4}}{16}\left(4 \ln \frac{d_{i j}}{\left(h_{1}-R\right)\left(h_{2}-R\right)}-1\right)+C_{0}^{1}, \\
& F_{D_{i j}}^{2}\left(d_{i j}\right)=\frac{C_{1}^{2} d_{i j}^{2}}{2}+\frac{C_{2}^{2} d_{i j}^{3}}{3}+\frac{C_{3}^{2} d_{i j}^{4}}{4}+C_{0}^{2} \\
& F_{D_{i j}}^{3}\left(d_{i j}\right)=\frac{C_{1}^{3} d_{i j}^{2}}{2}+\frac{C_{2}^{3} d_{i j}^{3}}{3}+\frac{C_{3}^{3} d_{i j}^{4}}{4}+\frac{C_{4}^{3} d_{i j}^{2}}{4}\left(2 \ln \frac{d_{i j}}{\left(h_{1}+R\right)\left(h_{2}+R\right)}-1\right)+\frac{C_{5}^{3} d_{i j}^{3}}{9}\left(3 \ln \frac{d_{i j}}{\left(h_{1}+R\right)\left(h_{2}+R\right)}-1\right)+\left(3 \ln \frac{d_{i j}}{\left(h_{1}+R\right)\left(h_{2}+R\right)}\right)+\frac{C_{6}^{3} d_{3}^{4}}{16}\left(4 \ln \frac{d_{i j}}{\left(h_{1}+R\right)\left(h_{2}+R\right)}-1\right)+C_{0}^{3}, \\
& C_{1}^{1}=\frac{9\left(h_{1}-R\right)\left(h_{2}-R\right)\left(3 h_{1} h_{2}+2 h_{1} R+2 h_{2} R+R^{2}\right)}{16 h_{1} h_{2} R^{6}}, \\
& C_{2}^{1}=-\frac{9\left(h_{1}+h_{2}\right)}{4 h_{1} h_{2} R^{5}} \\
& C_{3}^{1}=\frac{1}{16 h_{1} h_{2} R^{6}}\left(\frac{-27 h_{1}+9 R}{2 h_{1}-2 R}+\frac{-27 h_{2}+9 R}{2 h_{2}-2 R}\right), \\
& C_{4}^{1}=\frac{9\left(h_{1}^{2}-R^{2}\right)\left(h_{2}^{2}-R^{2}\right)}{16 h_{1} h_{2} R^{6}}, \\
& C_{5}^{1}=\frac{9}{4 R^{6}}, \\
& C_{6}^{1}=\frac{9}{16 h_{1} h_{2} R^{6}}, \\
& C_{0}^{1}=-\frac{\left(h_{1}-R\right)^{3}\left(h_{2}-R\right)^{3}\left(h_{1}+3 R\right)\left(h_{2}+3 R\right)}{256 h_{1} h_{2} R^{6}}, \\
& C_{1}^{2}=\frac{9}{16 h_{1} h_{2} R^{6}}\left(\left(h_{1}^{2}-R^{2}\right)\left(h_{2}^{2}-R^{2}\right) \ln \frac{\left(h_{1}+R\right)}{\left(h_{1}-R\right)}-2 R h_{1}\left(h_{2}^{2}-R^{2}\right)\right) \text {, } \\
& C_{2}^{2}=\frac{9}{4 R^{6}} \ln \frac{\left(h_{1}+R\right)}{\left(h_{2}-R\right)}-\frac{18}{4 h_{1} R^{5}}, \\
& C_{3}^{2}=\frac{1}{16 h_{1} h_{2} R^{6}}\left(\frac{9 R-27 h_{1}}{2 h_{1}-2 R}+\frac{9 R+27 h_{2}}{2 h_{2}+2 R}+9 \ln \frac{\left(h_{1}+R\right)}{\left(h_{1}-R\right)}\right), \\
& C_{0}^{2}=F_{D_{i j}}^{1}\left(\left(h_{1}+R\right)\left(h_{2}-R\right)\right)-\frac{C_{1}^{2}\left(h_{1}+R\right)^{2}\left(h_{2}-R\right)^{2}}{2}-\frac{C_{2}^{2}\left(h_{1}+R\right)^{3}\left(h_{2}-R\right)^{3}}{3}-\frac{C_{3}^{2}\left(h_{1}+R\right)^{4}\left(h_{2}-R\right)^{4}}{4}, \\
& C_{1}^{3}=-\frac{9\left(h_{1}+R\right)\left(h_{2}+R\right)\left(3 h_{1} h_{2}-2 h_{1} R-2 h_{2} R+R^{2}\right)}{16 h_{1} h_{2} R^{6}}, \\
& C_{2}^{3}=-\frac{9\left(h_{1}+h_{2}\right)}{4 h_{1} h_{2} R^{5}}, \\
& C_{3}^{3}=\frac{1}{16 h_{1} h_{2} R^{6}}\left(\frac{27 h_{1}+9 R}{2 h_{1}+2 R}+\frac{27 h_{2}+9 R}{2 h_{2}+2 R}\right), \\
& C_{4}^{3}=-\frac{9\left(h_{1}^{2}-R^{2}\right)\left(h_{2}^{2}-R^{2}\right)}{16 h_{1} h_{2} R^{6}}, \\
& C_{5}^{3}=-\frac{9}{4 R^{6}} \\
& C_{6}^{3}=-\frac{9}{16 h_{1} h_{2} R^{6}}, \\
& C_{0}^{3}=F_{D_{i j}}^{2}\left(\left(h_{1}-R\right)\left(h_{2}+R\right)\right)-\frac{C_{1}^{3}\left(h_{1}-R\right)^{2}\left(h_{2}+R\right)^{2}}{2}-\frac{C_{2}^{3}\left(h_{1}-R\right)^{3}\left(h_{2}+R\right)^{3}}{3}-\frac{C_{3}^{3}\left(h_{1}-R\right)^{4}\left(h_{2}+R\right)^{4}}{4} \\
& -\frac{C_{4}^{3} d_{i j}^{2}}{4}\left(2 \ln \frac{\left(h_{1}-R\right)}{\left(h_{1}+R\right)}-1\right)+\frac{C_{5}^{3} d_{i j}^{3}}{9}\left(3 \ln \frac{\left(h_{1}-R\right)}{\left(h_{1}+R\right)}-1\right)+\frac{C_{6}^{3} d_{i j}^{3}}{16}\left(3 \ln \frac{\left(h_{1}-R\right)}{\left(h_{1}+R\right)}-1\right) .
\end{aligned}
$$

Proof of Theorem 2. the proof of Theorem 2 is given in Appendix A.

\section{The Area of Interest Coverage of SCFNR}

According to equation (1), the larger the product of transmitter-target and target-receiver distance $\left\|S_{i T} P\right\|\left\|P S_{j R}\right\|$, the smaller the received SNR, and the probability of the $P$ detected by transmitter-receiver is smaller too. Conversely, the smaller the $\left\|S_{i T} P\right\|\left\|P S_{j R}\right\|$, the larger the probability will be.

So, we define point coverage of SCFNR for target detection. 
Definition 2. Given a threshold value $c$ and a point target $P$, if there exists a bistatic radar $T_{i} R_{j} \in \mathbf{T R}(i \neq j)$ and the product of transmitter-target and target-receiver distances in any time slot of orbital hyperperiod is satisfied

$$
\left\|S_{i T} P\right\|\left\|P S_{j R}\right\|<c,
$$

then the bistatic $\operatorname{radar} T_{i} R_{j} \in \mathrm{TR}$ can provide point coverage to point $P$.

For the sake of analysis, according to the idea of grid, the surveillance region is divided into grids with equal borders, the border length of the grid is able to be elected in accordance with the range resolution of radar. That is, in ECI coordinate, the surveillance region of interest A (see Figure 4) is encoded in accordance with horizontal encoding $g_{x}\left(1 \leq g_{x} \leq N_{x}\right)$ and vertical encoding $g_{y}\left(1 \leq g_{y} \leq N_{y}\right)$; the grid coordinate $\left(g_{x}, g_{y}\right)$ is denoted by $A_{g_{x} g_{y}}$. Thus, the region of interest $\boldsymbol{A}$ can be determined uniquely by all grids and expressed as follows:

$$
\mathbf{A}=\left\{A_{g_{x} g_{y}} \mid 1 \leq g_{x} \leq N_{x}, 1 \leq g_{y} \leq N_{y}\right\}
$$

So, for a bistatic radar $T_{i} R_{j} \in \mathbf{T R}$, SNR of each grid can be given as follows:

$$
\mathrm{SNR}_{g_{x} g_{y}}=\frac{K_{B}}{\left\|S_{i T} A_{g_{x} g_{y}}\right\|^{2}\left\|A_{g_{x} g_{y}} S_{j R}\right\|^{2}} .
$$

Let $\Gamma$ be the SNR threshold, then $c=\sqrt{K_{B} / \Gamma}$, and a grid target is covered by a bistatic radar $T_{i} R_{j} \in \mathbf{T R}$, if $\mathrm{SNR}_{g_{x} g_{\gamma}} \geq \Gamma$. Then, the definition of 0 - 1 grid coverage matrix on SCFNR can be described as follows:

Definition 3. For a bistatic radar $T_{i} R_{j} \in \mathrm{TR}$ in SCFNR, given $A_{g_{x} g_{y}} \in \mathbf{A}$, the $0-1$ grid coverage matrix is denoted by $\mathbf{U}_{i j}=\left[u_{i j, g_{x} g_{y}}\right]_{N_{x} \times N_{y}}$, where

$$
u_{i j, g_{x} g_{y}}= \begin{cases}1, & F_{D_{i j}}\left(\left\|S_{i T} A_{g_{x} g_{y}}\right\|\left\|A_{g_{x} g_{y}} S_{j R}\right\|\right) \leq F_{D_{i j}}(c), \\ 0, & \text { others. }\end{cases}
$$

If $u_{i j, g_{x} g_{y}}=1$ in equation (10), it indicates that the grid $A_{g_{x} g_{y}}$ can be covered by the bistatic radar $T_{i} R_{j} \in$ TR.

Additionally, to analyze the impact of transmitter-receiver pairs on coverage, $0-1$ pairing matrix, which describes the transmitter-receiver pairs selected in SCFNR, can be defined as follows.

Definition 4. For SCFNR, suppose the cardinalities both $\mathbf{T}$ and $\mathbf{R}$ are $N$. If $T_{i}$ and $R_{j}$ are selected as a bistatic radar, let $m_{i j}=1$; otherwise, $m_{i j}=0$. Then, $0-1$ pairing matrix of transmitter-receivers is denoted by $\mathbf{M}=\left[m_{i j}\right]_{N \times N}$.

Note that same grids may be covered by different bistatic radar pairs. Thus, based on Definitions 2 and 4, the introduction of cumulative coverage times $w_{g_{x} g_{y}}$ describes the coverage level of SCFNR at grid $A_{g_{x} g_{y}}$, that is,

$$
w_{g_{x} g_{y}}=\sum_{i=1}^{N} \sum_{j=1}^{N} m_{i j} u_{i j, g_{x} g_{y}} .
$$

As seen from equation (11), $w_{g_{x} g_{y}} \in\{0,1, \ldots, N\}$. On the basis of this, $K$-grid coverage matrix of SCNFR can be defined as follows.

Definition 5. For SCFNR, given a value $K(K \leq N)$, if the variable $c_{g_{x} g_{y}}$ is satisfied as

$$
c_{g_{x} g_{y}}= \begin{cases}1, & w_{g_{x} g_{y}} \geq K, \\ 0, & \text { otherwise }\end{cases}
$$

then the matrix $\mathbf{C} \in \mathbb{R}^{N_{x} \times N_{y}} ; \mathbf{C}=\left[c_{g_{x} g_{y}}\right]$ is called $K$-grid coverage matrix.

In equation (12), the total number of elements with 1 in $C$ represents the grid number satisfying $K$-grid coverage, and the total number of elements with 0 in $\mathbf{C}$ represents the grid number unsatisfying $K$-grid coverage, that is,

$$
g_{0}=N_{x} N_{y}-\sum_{g_{x}=1}^{N_{x}} \sum_{g_{y}=1}^{N_{y}} c_{g_{x} g_{y}}
$$

From the point of optimizing system, there is $g_{0} \longrightarrow 0$. If the values of $g_{0}$ approach 0 , then SCFNR can provide completely $K$-grid coverage to the region A; otherwise, SCFNR fails to provide $K$-grid coverage to the region $\mathbf{A}$. Therefore, the normalized $g_{0}$ is taken as $g_{1}$ to measure coverage performance of SCFNR, that is,

$$
g_{1}=1-\frac{1}{N_{x} N_{y}} \sum_{g_{x}=1}^{N_{x}} \sum_{g_{y}=1}^{N_{y}} c_{g_{x} g_{y}} .
$$

Also, using the radar equation and conditional probability, let $l_{i j}=\left\|S_{i T} A_{g_{x} g_{y}}\right\|\left\|A_{g_{x} g_{y}} S_{j R}\right\|$; the detection probability of bistatic radar $T_{i} R_{j} \in \mathbf{T R}$ to grid $A_{g_{x} g_{y}}$ is given by

$$
p_{i j, g_{x} g_{y}}=P_{r}\left\{d_{i j} \geq l_{i j} \mid d_{i j} \leq c\right\} \text {. }
$$

Thus, the detection probability of SCFNR radar to grid $A_{g_{x} g_{y}}$ is as follows:

$$
p_{g_{x} g_{y}}=1-\prod_{i=1}^{N} \prod_{j=1}^{N}\left(1-m_{i j} p_{i j, g_{x} g_{y}}\right) .
$$

For the sake of optimization analysis, the worst-case detection probability of all grids is taken as the second objective function to measure the region detection performance of SCFNR, that is,

$$
p_{\text {net }} \triangleq \min _{g_{x}, g_{y}}\left(p_{g_{x} g_{y}}\right)
$$

To sum up, in SCFNR coverage scenario, $K$-grid coverage and detection probability (e.g., $g_{1}$ and $p_{\text {net }}$ ) are functions of pairing matrix M. Therefore, pairing transmitter-receivers with minimum $g_{1}$ and maximum $p_{\text {net }}$ can be optimized as follows: 


$$
\begin{aligned}
& \min g_{1}(\mathbf{M})=\left(1-\frac{1}{N_{x} N_{y}} \sum_{g_{x}=1}^{N_{x}} \sum_{g_{y}=1}^{N_{y}} c_{g_{x} g_{y}}\right) \\
& \min g_{2}(\mathbf{M})=\left(1-p_{\text {net }}\right) \\
& \qquad C_{1}: \sum_{i=1}^{N} m_{i j}=1, \quad \forall j \in\{1, \ldots, N\}, \\
& \text { s.t. } \quad C_{2}: \sum_{j=1}^{N} m_{i j}=1, \quad \forall i \in\{1, \ldots, N\} .
\end{aligned}
$$

The constraints $C_{1}$ and $C_{2}$ denote that each selected transmitter or receiver can only be associated to one receiver or transmitter. This is actually one-to-one pairing problem [40].

\section{Algorithm Design}

Obviously, the problem described in equation (18) is a multiobjective optimization problem. Due to the conflicting nature of the two objectives, no solution optimizing all objective functions simultaneously exists in general. Instead, balance among objective functions is taken into account, which is called trade-off analysis in multiobjective optimization, i.e., Pareto optimal solutions [41]. The basic idea is based on a distance measure to determine the solution near by the ideal solution. Here, the weighted Lp norm is taken as the distance measure, that is,

$$
g_{\mathbf{M}}=\left[\sum_{i_{b}=1}^{2} \zeta_{i_{b}}\left(g_{i_{b}}-g_{i_{b}}^{*}\right)^{p}\right]^{1 / p}
$$

where $g_{i_{b}}^{*}$ is the ideal value of $i_{b}$-th goal, and $g_{1}^{*}=g_{2}^{*}=0$, and $\zeta_{i_{b}}$ is the weight factor of the $i_{b}$-th goal.

The problem described in equation (19) is a combinatorial optimization problem. For this problem, particle swarm optimization (PSO) has been proved as an effective tool [42-44]. PSO is based on the behavior of birds flocking
[45]. Each particle represents a potential solution to optimization task and all particles fly in the search space to find the optimal solution. But, its solution does not satisfy the constraints $C_{1}$ and $C_{2}$. As mentioned before, the constraints $C_{1}$ and $C_{2}$ described in equation (18) are a one-to-one paring problem, which can be solved using Hungarian algorithm (HA) [40]. As a combinatorial optimization method, HA can finish the one-to-one paring task in polynomial time. Therefore, the PSO-HA integrated PSO with HA is proposed. The outline of PSO-HA is given as follows.

\section{Step 1. Initialization.}

Suppose swarm size is $L$, particle is $1(1 \leq l \leq L)$, the maximum number of iterations is $T_{\max }$, iteration time is $t\left(1 \leq t \leq T_{\max }\right)$, acceleration factors are $c_{1}$ and $c_{2}$, and the position and velocity of $l$-th particle are $\bar{M}_{l}$ and $\mathbf{v}_{l}$, respectively. Let $t=1$, and set the parameter values: $L, T_{\max }$, $\bar{M}_{l}(t), \mathbf{v}_{l}(t), c_{1}$, and $c_{2}$.

As a note, $\bar{M}_{l}$ and $\mathbf{v}_{l}$ are both $N \times N$ matrixes, in which each element of matrixes is generated randomly. Here, each element of $\bar{M}_{l}$ and $\mathbf{v}_{l}$ is set in the range $[0,1]$ and $[-0.5,0.5]$, respectively.

Step 2. Update position $\bar{M}_{l}$ by using PSO.

In each iteration, $\bar{M}_{l}$ and $\mathbf{v}_{l}$ are updated as follows:

$$
\begin{aligned}
\mathbf{v}_{l}(t+1) & =\omega(t) \times \mathbf{v}_{l}(t)+c_{1} r_{1}(t)\left(\boldsymbol{\rho}_{l}(t)-\bar{M}_{l}(t)\right)+c_{1} r_{1}(t)\left(\boldsymbol{\rho}_{g}(t)-\bar{M}_{l}(t)\right), \\
\bar{M}_{l}(t+1) & =\bar{M}_{l}(t)+\mathbf{v}_{l}(t+1),
\end{aligned}
$$

where $\rho_{l}$ is the current position of $l$-th particle, $\rho_{g}$ is the best position of all particles it has visited so far, $\omega(t)$ is the inertia weight which decreases with iteration time as $\omega(t)=0.9-$ $0.5 \times\left(t / T_{\max }\right)$ [46], and $r_{1}(t)$ and $r_{2}(t)$ are random independent variables in the range $[0,1]$.

Step 3. Generate one-to-one paring matrix $\mathbf{M}_{l}$ by using HA.

The updating result $\bar{M}_{l}$ is taken as the cost matrix of $l$-th particle in HA, and then the optimization problem on oneto-one paring is formulated as follows:

$$
\begin{array}{ll}
\min & \sum_{i=1}^{N} \sum_{j=1}^{N} \bar{m}_{i j}^{l} m_{i j}^{l} \\
& C_{1}: \sum_{i=1}^{N} m_{i j}=1, \quad \forall j \in\{1, \ldots, N\}, \\
\text { s.t. } & C_{2}: \sum_{j=1}^{N} m_{i j}=1, \quad \forall i \in\{1, \ldots, N\},
\end{array}
$$

where $\bar{m}_{i j}^{l}$ is the element of matrix $\bar{M}_{l}, m_{i j}^{l}$ is the element of matrix $\mathbf{M}_{l}$, and $m_{i j}^{l}$ is either 0 or 1 .

Note that the paring matrix $\mathbf{M}_{l}$, which satisfies constraints $C_{1}$ and $C_{2}$, is obtained by using HA. 
Step 4. Calculate the fitness function $g_{\mathrm{M}}^{l}$ of $l$-th particle and determine the best solution $\mathbf{M}_{g}$ (i.e., optimal paring matrix).

Taking the objective function, i.e., equation (19), as the fitness function of $l$-th particle (denoted by $g_{M}^{l}$ ), the current paring matrix $\mathbf{M}_{l}$ of $l$-th particle and optimal paring matrix $\mathbf{M}_{g}$ of all particles are updated as follows:

$$
\begin{aligned}
& \mathbf{M}_{l}(t+1)= \begin{cases}\mathbf{M}_{l}(t+1), & \text { if } g_{\mathbf{M}}^{l}(t+1) \leq g_{\mathbf{M}}^{l}(t), \\
\mathbf{M}_{l}(t), & \text { others, }\end{cases} \\
& \mathbf{M}_{g}(t+1)=\mathbf{M}_{l}(t+1) \text { when } \min g_{\mathbf{M}}^{l}(t+1) 1 \leq l \leq L .
\end{aligned}
$$

Step 5. If $t \leq T_{\max }$, then increment $t$ and go to Step 2; otherwise, end.

\section{Simulation Analysis}

In order to simulate and analyze multiobjective pairing optimization, i.e., coverage and detection performances of SCFNR, in time slot of the orbital hyperperiod, we establish the SCFNR scenario by STK (Satellite Tool Kit) first. Then, we use PSO-HA to find optimal pairing matrix in Windows 10 and MATLAB R2017b environment. At the same time, area of interest coverage and detection probability are analyzed numerically.

\subsection{Parameters Setting}

6.1.1. Orbital Elements in SCFNR. Suppose SCFNR is composed of 4 pairs of homogeneous bistatic radars. Let $\mathbf{T}=\left\{T_{1}, T_{2}, T_{3}, T_{4}\right\}, \quad \mathbf{R}=\left\{R_{1}, R_{2}, R_{3}, R_{4}\right\}, m=30 \mathrm{~km}, \quad$ and $M=850 \mathrm{~km}$. According to the orbit design of cluster flight spacecraft proposed in [10], all near circular orbital elements of SCFNR are listed in Table 1.
According to Table 1, all orbital periods can be calculated and are approximated as 6310 seconds using STK, so we believe the orbital hyperperiods of the SCFNR are also 6310 s. In addition, as shown in Figure 5, we can also calculate all relative distances between transmitters and receivers in 172 days by STK. It is observed that the relative distance between any transmitter-receiver always remains below $850 \mathrm{~km}$ and above $30 \mathrm{~km}$.

6.1.2. The Target Grid and Other Parameters. Suppose that the longitude and latitude of surveillance regions are in the range [0, 0.07865345] ( $\mathrm{rad})$ and [0, 0.07865345] (rad), respectively. That is, surveillance region is set as square with the size of $500 \times 500 \mathrm{~km}$ on the earth surface. The region with longitude and latitude can be divided into $N_{x} \times N_{y}$ grids. Let $N_{x}=100$ and $N_{y}=100$. So, according to coordinate transforming relations between spherical coordinates and rectangular coordinates, each grid can be computed in the ECI coordinate.

For radar equation and PSO-HA, the parameters are listed in Table 2. In this case, the distribution function of $d_{i j}$ for SCFNR in equation (5) can be calculated, as presented in equation (23). At the same time, as shown in Figure 6, we give the curve of distribution function of $d_{i j}$ for SCFNR.

$$
F_{D_{i j}}\left(d_{i j}\right)= \begin{cases}0, & d_{i j}<7.04878 \times 10^{5}, \\ F_{1}\left(d_{i j}\right), & 7.04878 \times 10^{5} \leq d_{i j}<1.04910 \times 10^{6}, \\ F_{2}\left(d_{i j}\right), & 1.04910 \times 10^{6} \leq d_{i j} \leq 1.56142 \times 10^{6} \\ 1, & \text { otherwise, }\end{cases}
$$

where

$$
\begin{aligned}
& F_{1}\left(d_{i j}\right) \approx 8.30169 \times 10^{-9} \times d_{i j}^{2}-7.33464 \times 10^{-15} \times \\
& d_{i j}^{3}-6.49148 \times 10^{-21} \times d_{i j}^{4}+3.82234 \times 10^{-9} \times d_{i j}^{2} \times \log \left(\frac{d_{i j}}{7.04878 \times 10^{5}}\right)+1.01050 \times 10^{-14} \times d_{i j}^{3} \times \\
& \quad \log \left(\frac{d_{i j}}{7.04878 \times 10^{5}}\right)+1.73646 \times 10^{-21} \times d_{i j}^{4} \times \log \left(\frac{d_{i j}}{7.04878 \times 10^{5}}\right)+46.52592 \\
& F_{2}\left(d_{i j}\right) \approx-1.14225 \times 10^{-8} \times d_{i j}^{2}-5.97942 \times 10^{-16} \times d_{i j}^{3}+5.07373 \times 10^{-21} \times d_{i j}^{4}-3.82234 \times 10^{-9} \times d_{i j}^{2} \times \\
& \log \left(\frac{d_{i j}}{1.56143 \times 10^{6}}\right)-1.01050 \times 10^{-14} \times d_{i j}^{3} \times \log \left(\frac{d_{i j}}{1.56143 \times 10^{6}}\right)-1.73646 \times 10^{-21} \times d_{i j}^{4} \times \log \left(\frac{d_{i j}}{1.56143 \times 10^{6}}\right)-32.88394
\end{aligned}
$$


TABLE 1: All near circular orbital elements of SCFNR.

\begin{tabular}{lcccccc}
\hline Parameter & $\begin{array}{c}\text { Semimajor axis } \\
(\mathrm{km})\end{array}$ & $\begin{array}{c}\text { Eccentricity } \\
(\mathrm{deg})\end{array}$ & $\begin{array}{c}\text { Inclination } \\
(\mathrm{deg})\end{array}$ & $\begin{array}{c}\text { Argument of perigee } \\
(\mathrm{deg})\end{array}$ & $\begin{array}{c}\text { True anomaly } \\
(\mathrm{deg})\end{array}$ & $\begin{array}{c}\text { Right ascension of ascending } \\
\text { node }(\mathrm{deg})\end{array}$ \\
\hline$T_{1}$ & 7378.14 & 0.02 & 35 & 0.00000 & 0.00000 & 0.00000 \\
$T_{2}$ & 7378.14 & 0.02 & 35 & 0.00163 & 1.13947 & 3.38820 \\
$T_{3}$ & 7378.14 & 0.02 & 35 & 0.00068 & 0.47630 & 1.82400 \\
$T_{4}$ & 7378.14 & 0.02 & 35 & 359.997 & -2.1650 & 1.70983 \\
$R_{1}$ & 7378.14 & 0.02 & 35 & 0.00106 & 2.50602 & 2.50602 \\
$R_{2}$ & 7378.14 & 0.02 & 35 & 0.00022 & 0.15383 & 1.08022 \\
$R_{3}$ & 7378.14 & 0.02 & 35 & 359.999 & -0.79763 & 4.02245 \\
$R_{4}$ & 7378.14 & 0.02 & 35 & 0.00344 & 2.39289 & -1.18115 \\
\hline
\end{tabular}
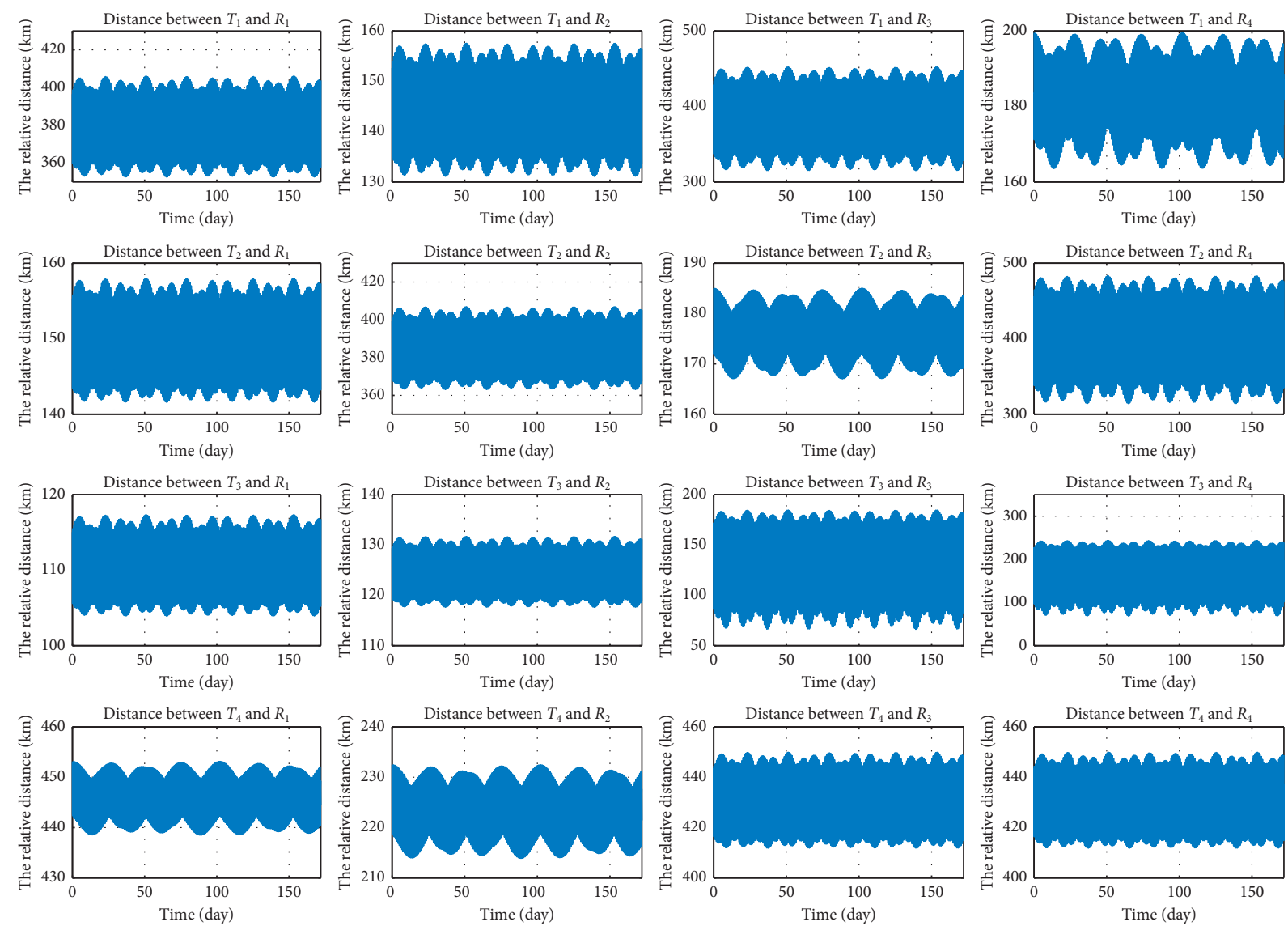

FIGURE 5: The relative distance between any transmitter-receiver within 172 days.

TABle 2: Parameter setting.

\begin{tabular}{lr}
\hline Parameter & Value \\
\hline$K_{\mathrm{B}}$ & $45 \mathrm{M}^{4}$ \\
$\Gamma$ & $12.5 \mathrm{~dB}$ \\
$h_{1}$ & $1044.5702 \mathrm{~km}$ \\
$h_{2}$ & $1044.5702 \mathrm{~km}$ \\
$c$ & $1.14932 \times 10^{6}$ \\
$F_{D_{i j}}(c)$ & 0.62269 \\
$L$ & 10 \\
$T_{\max }$ & 100 \\
$P$ & 2 \\
$c_{1}$ & 1.49445 \\
$c_{2}$ & 1.49445 \\
$R$ & $205 \mathrm{~km}$ \\
\hline
\end{tabular}


TABLE 3: $g_{M}$ for 24 cases of pairing schemes.

\begin{tabular}{|c|c|c|c|}
\hline Paring scheme & $\begin{array}{l}\zeta_{1}=0.7 \\
\zeta_{2}=0.3\end{array}$ & $\begin{array}{l}\zeta_{1}=0.5 \\
\zeta_{2}=0.5\end{array}$ & $\begin{array}{l}\zeta_{1}=0.3 \\
\zeta_{2}=0.7\end{array}$ \\
\hline$T_{1} R_{1}, T_{2} R_{2}, T_{3} R_{3}, T_{4} R_{4}$ & 0.15540 & 0.25500 & 0.35597 \\
\hline$T_{1} R_{1}, T_{2} R_{2}, T_{3} R_{4}, T_{4} R_{3}$ & 0.16469 & 0.24026 & 0.32681 \\
\hline$T_{1} R_{1}, T_{2} R_{3}, T_{3} R_{4}, T_{4} R_{2}$ & 0.13368 & 0.19492 & 0.26509 \\
\hline$T_{1} R_{1}, T_{2} R_{3}, T_{3} R_{2}, T_{4} R_{4}$ & 0.15069 & 0.24229 & 0.33688 \\
\hline$T_{1} R_{1}, T_{2} R_{4}, T_{3} R_{2}, T_{4} R_{3}$ & 0.15501 & 0.22166 & 0.29994 \\
\hline$T_{1} R_{1}, T_{2} R_{4}, T_{3} R_{3}, T_{4} R_{2}$ & 0.12747 & 0.19374 & 0.26614 \\
\hline$T_{1} R_{2}, T_{2} R_{1}, T_{3} R_{3}, T_{4} R_{4}$ & 0.09853 & 0.15754 & 0.21879 \\
\hline$T_{1} R_{2}, T_{2} R_{1}, T_{3} R_{4}, T_{4} R_{3}$ & 0.11394 & 0.13584 & 0.17271 \\
\hline$T_{1} R_{2}, T_{2} R_{4}, T_{3} R_{3}, T_{4} R_{1}$ & 0.11183 & 0.18308 & 0.25544 \\
\hline$T_{1} R_{2}, T_{2} R_{4}, T_{3} R_{1}, T_{4} R_{3}$ & 0.11765 & 0.14436 & 0.18576 \\
\hline$T_{1} R_{2}, T_{2} R_{3}, T_{3} R_{1}, T_{4} R_{4}$ & 0.09844 & 0.15016 & 0.20646 \\
\hline$T_{1} R_{2}, T_{2} R_{3}, T_{3} R_{4}, T_{4} R_{1}$ & 0.11829 & 0.18977 & 0.26374 \\
\hline$T_{1} R_{3}, T_{2} R_{1}, T_{3} R_{2}, T_{4} R_{4}$ & 0.10855 & 0.17487 & 0.24324 \\
\hline$T_{1} R_{3}, T_{2} R_{1}, T_{3} R_{4}, T_{4} R_{2}$ & 0.10459 & 0.15033 & 0.20370 \\
\hline$T_{1} R_{3}, T_{2} R_{2}, T_{3} R_{4}, T_{4} R_{1}$ & 0.14080 & 0.23239 & 0.32475 \\
\hline$T_{1} R_{3}, T_{2} R_{2}, T_{3} R_{1}, T_{4} R_{4}$ & 0.12640 & 0.20594 & 0.28708 \\
\hline$T_{1} R_{3}, T_{2} R_{4}, T_{3} R_{1}, T_{4} R_{2}$ & 0.13627 & 0.21010 & 0.28956 \\
\hline$T_{1} R_{3}, T_{2} R_{4}, T_{3} R_{2}, T_{4} R_{1}$ & 0.12935 & 0.21310 & 0.29770 \\
\hline$T_{1} R_{4}, T_{2} R_{3}, T_{3} R_{2}, T_{4} R_{1}$ & 0.14810 & 0.24148 & 0.33667 \\
\hline$T_{1} R_{4}, T_{2} R_{3}, T_{3} R_{1}, T_{4} R_{2}$ & 0.11359 & 0.15552 & 0.20788 \\
\hline$T_{1} R_{4}, T_{2} R_{1}, T_{3} R_{2}, T_{4} R_{3}$ & 0.12759 & 0.16616 & 0.21857 \\
\hline$T_{1} R_{4}, T_{2} R_{1}, T_{3} R_{3}, T_{4} R_{2}$ & 0.09847 & 0.13839 & 0.18638 \\
\hline$T_{1} R_{4}, T_{2} R_{2}, T_{3} R_{1}, T_{4} R_{3}$ & 0.13989 & 0.19168 & 0.25627 \\
\hline$T_{1} R_{4}, T_{2} R_{2}, T_{3} R_{3}, T_{4} R_{1}$ & 0.15399 & 0.25457 & 0.35585 \\
\hline
\end{tabular}

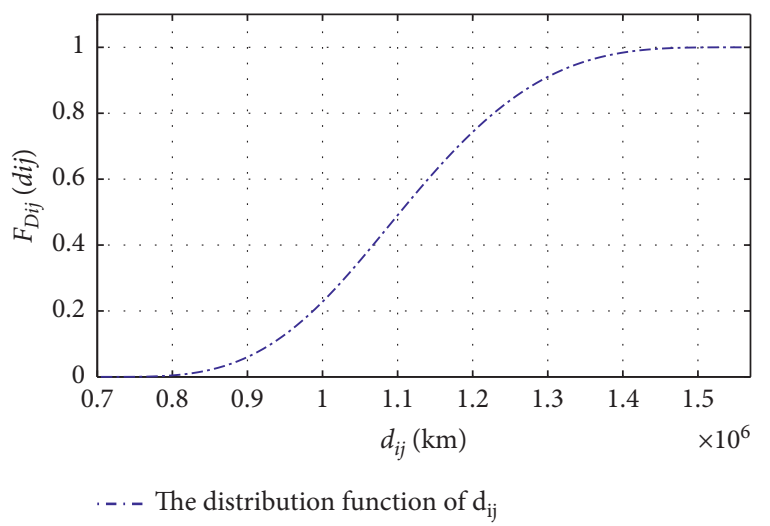

Figure 6: The distance distribution function (equation (23)) associated with $d_{i j}$ in SCFNR.

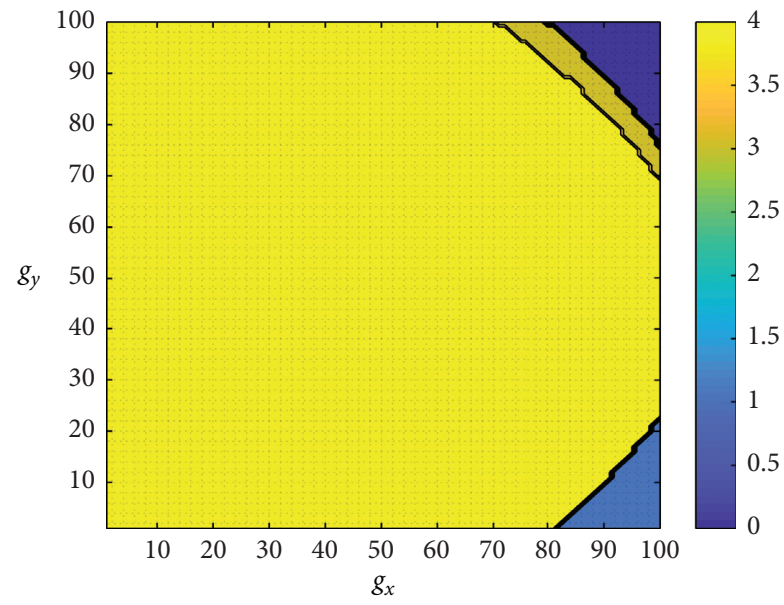

(a)

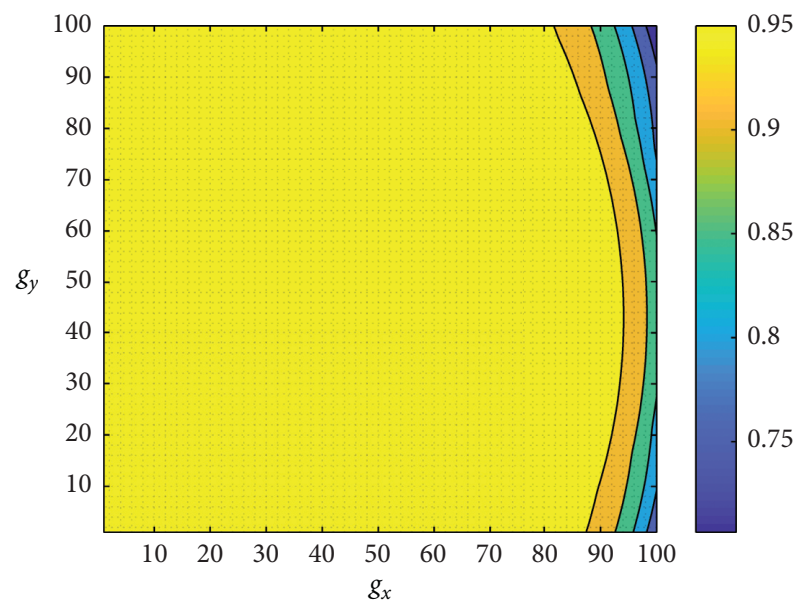

(b)

FIgURe 7: The distributions of coverage level and detection probability with $\zeta_{1}=0.7, \zeta_{2}=0.3$, and $\Gamma=12.5 \mathrm{~dB}$ in grids. (a) $w_{g_{x} g_{y}}$. (b) $p_{g_{x} g_{y}}$. 


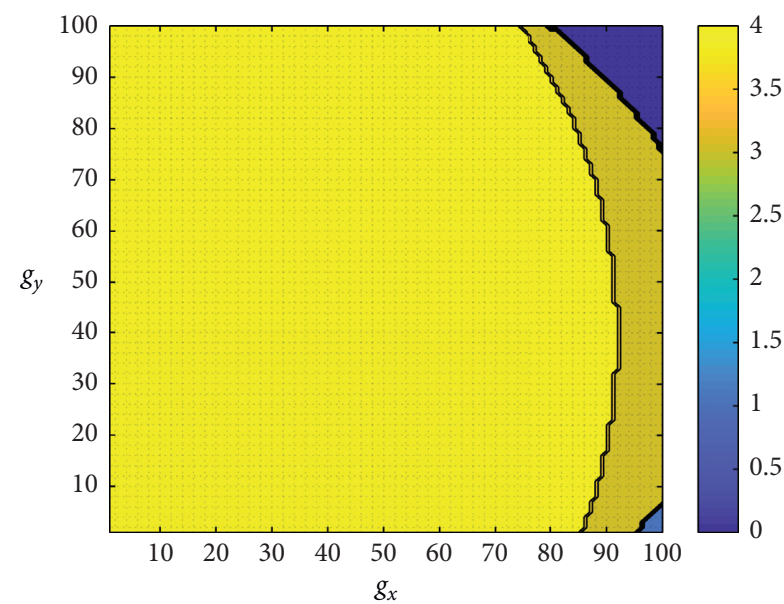

(a)

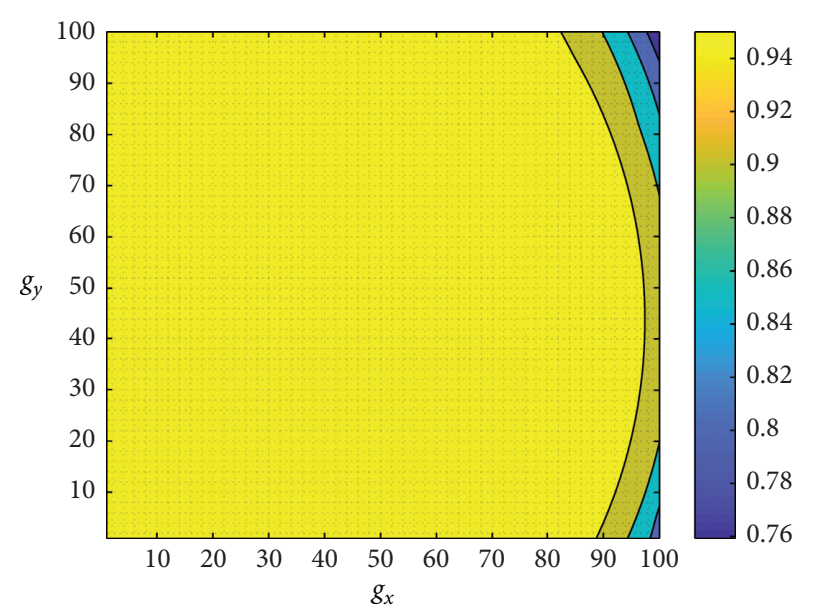

(b)

FIGURE 8: The distributions of coverage level and detection probability with $\zeta_{1}=0.5, \zeta_{2}=0.5$, and $\Gamma=12.5 \mathrm{~dB}$ in grids. (a) $w_{g_{x} g_{y}}$. $(\mathrm{b}) p_{g_{x} g_{y}}$.

\subsection{Numerical Result and Analysis}

\subsubsection{Transmitter-Receiver Pairing Scheme.}

Case1: $g_{M}$ with different weight values in the slot 1 for the 1 st orbital hyperperiod.

Considering three conditions, i.e., $\zeta_{1}>\zeta_{2}, \zeta_{1}=\zeta_{2}$, and $\zeta_{1}<\zeta_{2}$, we calculate the optimal pairing matrix in the same slot of its orbital hyperperiod under the same simulation environment as described in Section 6.1.

Let $\zeta_{1}=0.7$ and $\zeta_{2}=0.3$; the optimal pairing matrix is given as follows:

$$
M_{g}=\left[\begin{array}{llll}
0 & 1 & 0 & 0 \\
0 & 0 & 1 & 0 \\
1 & 0 & 0 & 0 \\
0 & 0 & 0 & 1
\end{array}\right] .
$$

Equation (25) indicates that optimal pairing scheme of bistatic radars is $T_{1} R_{2}, T_{2} R_{3}, T_{3} R_{1}, T_{4} R_{4}$, and $g_{\mathrm{M}}=0.098349$ with $g_{1}=0.06270$ and $g_{2}=0.29371$.

Let $\zeta_{1}=0.5$ and $\zeta_{2}=0.5$; the optimal pairing matrix is given as follows:

$$
\mathbf{M}_{g}=\left[\begin{array}{llll}
0 & 1 & 0 & 0 \\
1 & 0 & 0 & 0 \\
0 & 0 & 0 & 1 \\
0 & 0 & 1 & 0
\end{array}\right] .
$$

Equation (26) indicates that optimal pairing scheme of bistatic radars is $T_{1} R_{2}, T_{2} R_{1}, T_{3} R_{4}, T_{4} R_{3}$, and $g_{\mathrm{M}}=0.135841$ with $g_{1}=0.1259$ and $g_{2}=0.24075$.

Let $\zeta_{1}=0.3$ and $\zeta_{2}=0.7$; the optimal pairing matrix is given as follows:

$$
\mathbf{M}_{g}=\left[\begin{array}{llll}
0 & 1 & 0 & 0 \\
1 & 0 & 0 & 0 \\
0 & 0 & 0 & 1 \\
0 & 0 & 1 & 0
\end{array}\right] .
$$

Equation (27) indicates that the optimal pairing scheme of bistatic radars is $T_{1} R_{2}, T_{2} R_{1}, T_{3} R_{4}, T_{4} R_{3}$, and $g_{\mathrm{M}}=$ 0.172705 with $g_{1}=0.1259$ and $g_{2}=0.24075$.

For the sake of comparison, Table 3 lists all pairing schemes for 4 pairs of homogeneous bistatic radars, i.e., 24 cases of pairing schemes and corresponding $g_{\mathbf{M}}$ using the same parameters.

As shown in Table 3, we mark the $g_{M}$ in bold font. It can be seen that, under conditions of same weight values, the corresponding paring schemes are consistent with equations (25) and (26), respectively. Therefore, PSO$\mathrm{HA}$ is effective and reasonable.

Case 2: $g_{\mathrm{M}}$ with different weight values in the slots 1 and 2 of different orbital hyperperiods.

When $\zeta_{1}=0.7$ and $\zeta_{2}=0.3$, the optimal pairing scheme and corresponding $g_{\mathrm{M}}$ can be listed in Table 4, and when $\zeta_{1}=0.5$ and $\zeta_{2}=0.5$, the optimal pairing scheme and corresponding $g_{\mathbf{M}}$ can be listed in Table 5 .

As Tables 4 and 5 show, the optimal pairing schemes are various in different slots of different orbital hyperperiods, and $\zeta_{2}=0.3$ is different as well. From this result, we conclude that geometric topology of SCFNR with high dynamic and random leads to optimal pairing scheme with dynamic and random.

\subsubsection{Coverage Level and Detection Probability.}

Case 1: when $\Gamma=12.5 \mathrm{~dB}$,

Using the optimal pairing matrices given by equations (25)-(27), we calculate the corresponding distributions of coverage level and detection probability as shown in Figures 7 and 8 . The coverage level and detection probability corresponding to equations (26) and (27) are the same due to the same optimal pairing matrices in equations (26) and (27). So, we only need to give the two distributions with $\zeta_{1}=0.7, \zeta_{2}=0.3$ and $\zeta_{1}=0.5$, $\zeta_{2}=0.5$, respectively. 
TABLE 4: Optimal pairing scheme and corresponding $g_{\mathrm{M}}$ with $\zeta_{1}=0.7$ and $\zeta_{2}=0.3$.

\begin{tabular}{lccc}
\hline Orbital hyperperiod & Time slot & Optional pairing scheme & $g_{\mathbf{M}}$ \\
\hline \multirow{2}{*}{ The 2nd orbital hyperperiod } & 1 & $T_{1} R_{2}, T_{2} R_{1}, T_{3} R_{3}, T_{4} R_{4}$ & 0.063586 \\
& 2 & $T_{1} R_{1}, T_{2} R_{2}, T_{3} R_{3}, T_{4} R_{4}$ & 0.139745 \\
\hline \multirow{2}{*}{ The 3th orbital hyperperiod } & 1 & $T_{1} R_{2}, T_{2} R_{1}, T_{3} R_{3}, T_{4} R_{4}$ & 0.047843 \\
& 2 & $T_{1} R_{3}, T_{2} R_{4}, T_{3} R_{1}, T_{4} R_{3}$ & 0.123073 \\
The 12th orbital hyperperiod & 1 & $T_{1} R_{2}, T_{2} R_{3}, T_{3} R_{4}, T_{4} R_{1}$ & 0.009715 \\
& 2 & $T_{1} R_{1}, T_{2} R_{4}, T_{3} R_{3}, T_{4} R_{2}$ & 0.198599 \\
The 16th orbital hyperperiod & 1 & $T_{1} R_{2}, T_{2} R_{3}, T_{3} R_{4}, T_{4} R_{1}$ & 0.068867 \\
& 2 & $T_{1} R_{1}, T_{2} R_{3}, T_{3} R_{4}, T_{4} R_{2}$ & 0.295543 \\
\hline
\end{tabular}

TABLE 5: Optimal pairing scheme and corresponding $g_{\mathrm{M}}$ with $\zeta_{1}=0.5$ and $\zeta_{2}=0.5$.

\begin{tabular}{lccr}
\hline Orbital hyperperiod & Time slot & Optional pairing scheme & $g_{\mathbf{M}}$ \\
\hline \multirow{2}{*}{ The 2nd orbital hyperperiod } & 1 & $T_{1} R_{3}, T_{2} R_{1}, T_{3} R_{4}, T_{4} R_{2}$ & 0.098536 \\
& 2 & $T_{1} R_{2}, T_{2} R_{1}, T_{3} R_{3}, T_{4} R_{4}$ & 0.072687 \\
& 1 & $T_{1} R_{3}, T_{2} R_{4}, T_{3} R_{2}, T_{4} R_{1}$ & 0.156137 \\
The 3th orbital hyperperiod & 2 & $T_{1} R_{2}, T_{2} R_{4}, T_{3} R_{1}, T_{4} R_{3}$ & 0.156137 \\
& 1 & $T_{1} R_{2}, T_{2} R_{3}, T_{3} R_{4}, T_{4} R_{1}$ & 0.015381 \\
The 12th orbital hyperperiod & 2 & $T_{1} R_{1}, T_{2} R_{4}, T_{3} R_{3}, T_{4} R_{2}$ & 0.251995 \\
& 1 & $T_{1} R_{4}, T_{2} R_{3}, T_{3} R_{1}, T_{4} R_{2}$ & 0.090544 \\
The 16th orbital hyperperiod & 2 & $T_{1} R_{1}, T_{2} R_{3}, T_{3} R_{4}, T_{4} R_{2}$ & 0.380717 \\
\hline
\end{tabular}

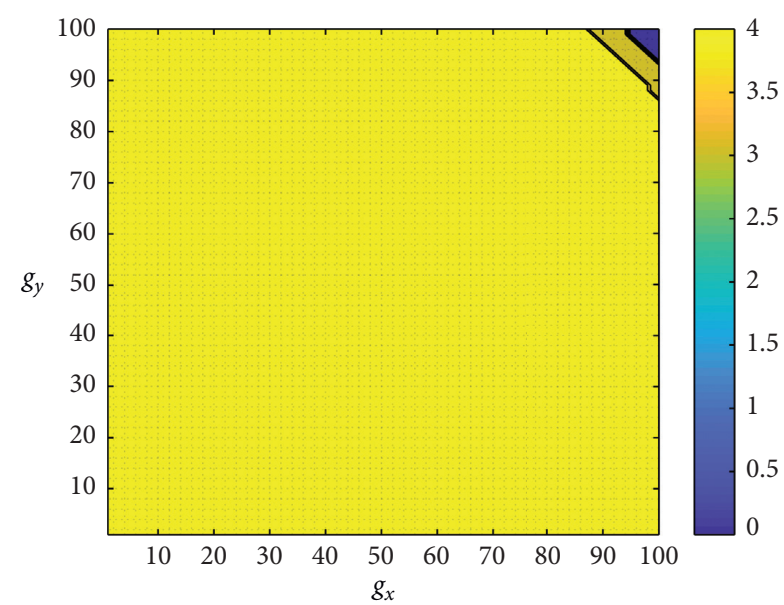

(a)

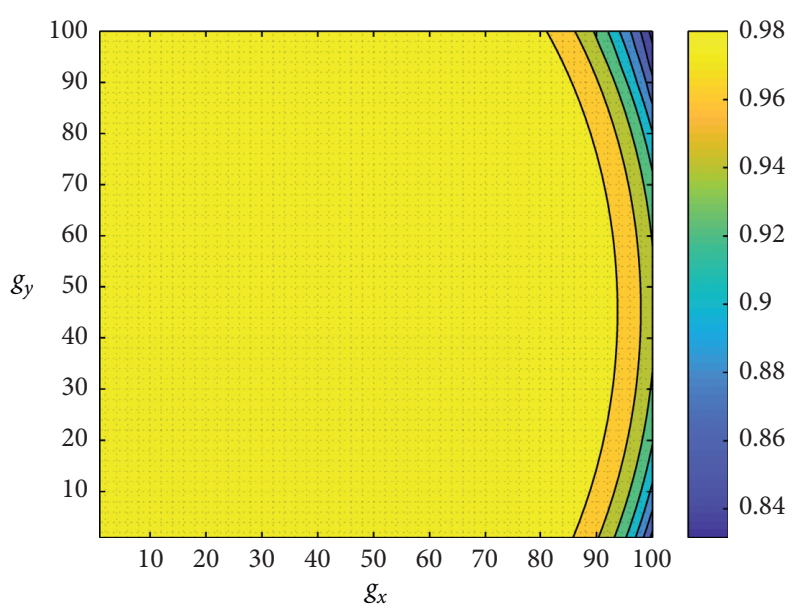

(b)

FIGURE 9: The distributions of coverage level and detection probability with $\zeta_{1}=0.7, \zeta_{2}=0.3$, and $\Gamma=12.0 \mathrm{~dB}$ in grids. (a) $w_{g_{x} g_{y}}$. $(\mathrm{b}) p_{g_{x} g_{y}}$.

In Figures 7 and 8, it is observed that either the distribution of coverage level or the distribution of detection probability is roughly the same. Besides, the higher the coverage level, the higher the detection probability.

Case 2: when $\Gamma=12.0 \mathrm{~dB}$,

In this case, keeping other parameters unchanged, the distributions of coverage level and detection probability are shown in Figures 9-11.

In Figures 9-11, it is observed that the three distributions of coverage level and detection probability are roughly the same with different weight values. However, there is considerable difference between the distributions with $\Gamma=12.5 \mathrm{~dB}$ and $\Gamma=12.0 \mathrm{~dB}$, and we find that the SNR threshold has a great influence on coverage level and detection probability in SCFNR. We also observe that the smaller the threshold $\Gamma$, the larger the coverage level and detection probability. These show that the proposed PSO-HA, coverage, and detection probability model are reasonable and effective, especially for coverage and detection performance measured by distance function.

In addition, using PSO-HA to solve optimal pairing matrix, considering three weight values, we give the relationship between iteration and $g_{M}$ in slot 1 for the 1 st orbital hyperperiod. As shown in Figure 12, the iterative process has good convergence. 


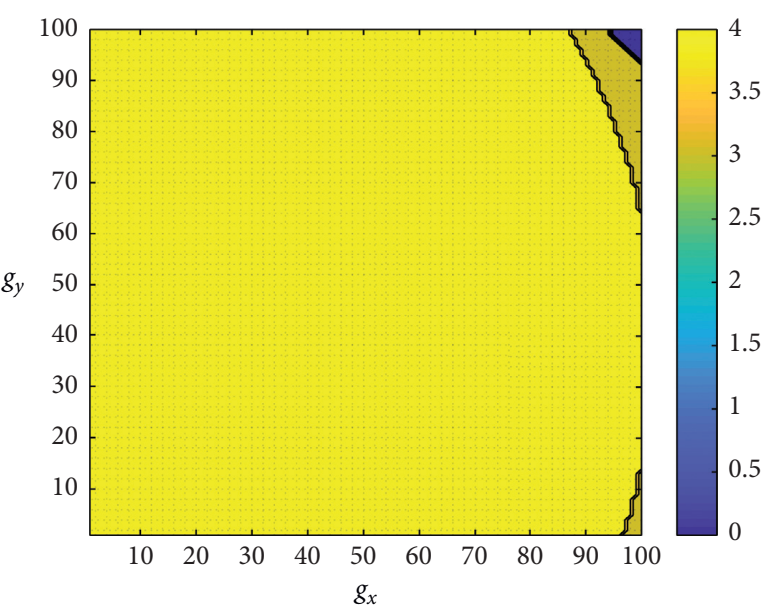

(a)

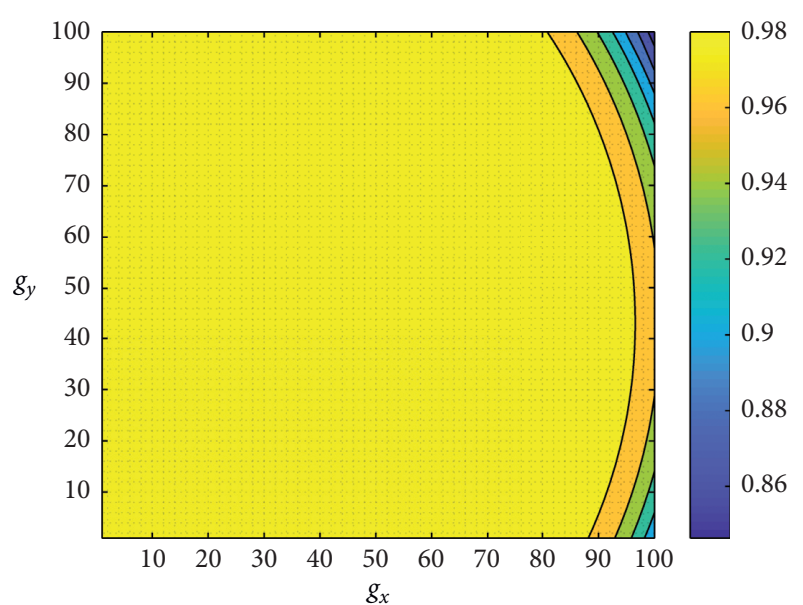

(b)

FIGURE 10: The distributions of coverage level and detection probability with $\zeta_{1}=0.5, \zeta_{2}=0.5$, and $\Gamma=12.0 \mathrm{~dB}$ in grids. (a) $w_{g_{x} g_{y}}$. $(\mathrm{b}) p_{g_{x} g_{y}}$.

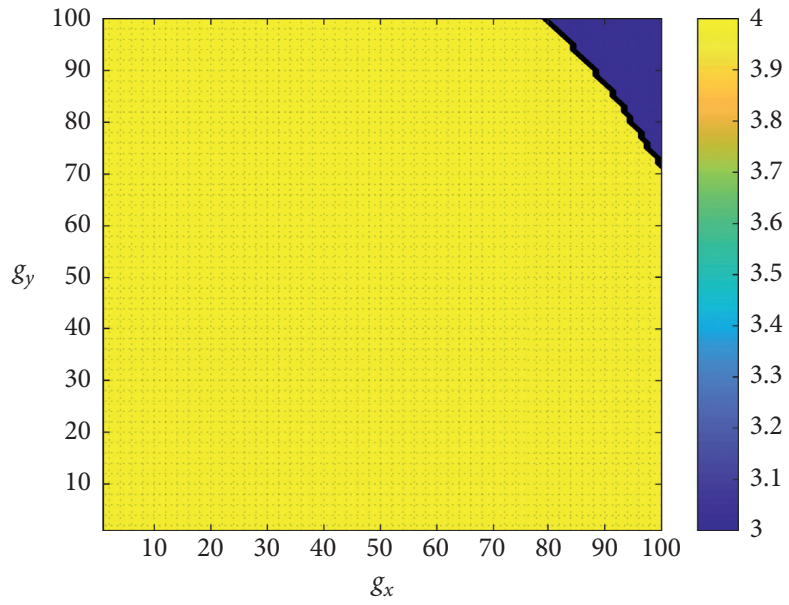

(a)

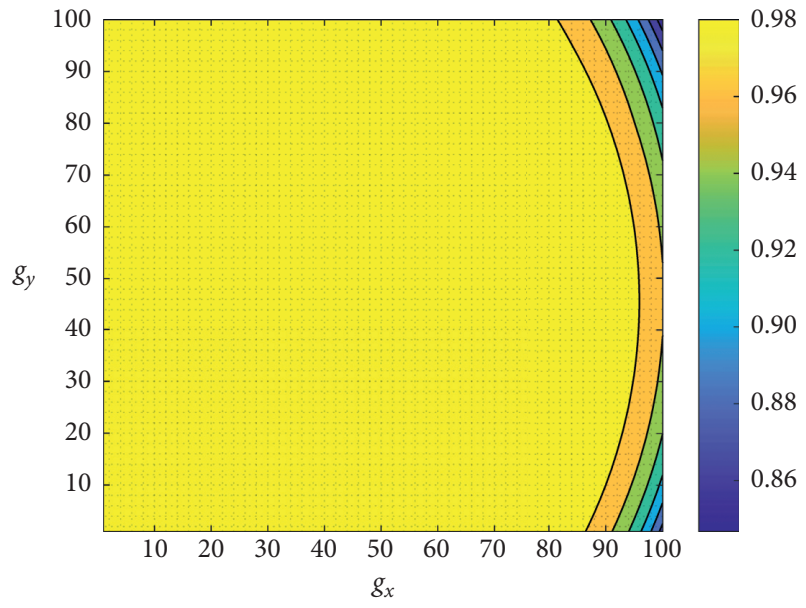

(b)

FIGURE 11: The distributions of coverage level and detection probability with $\zeta_{1}=0.3, \zeta_{2}=0.7$, and $\Gamma=12.0 \mathrm{~dB}$ in grids. (a) $w_{g_{x} g_{y}}$. (b) $p_{g_{x} g_{y}}$.

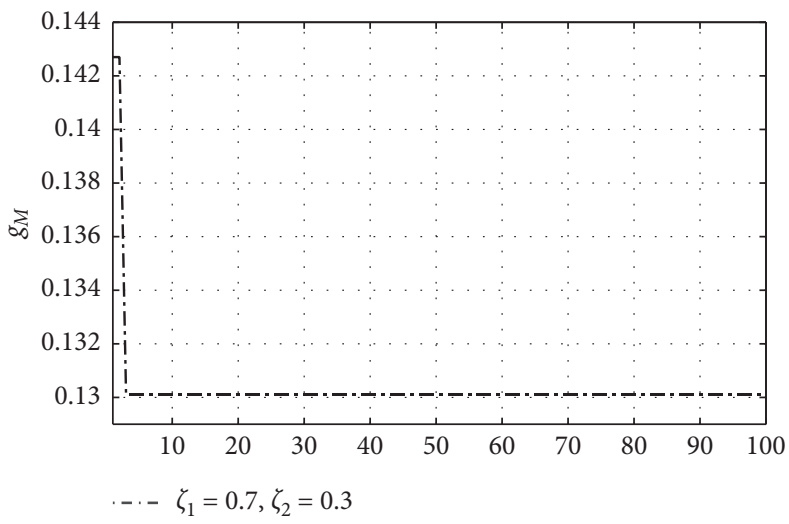

(a)

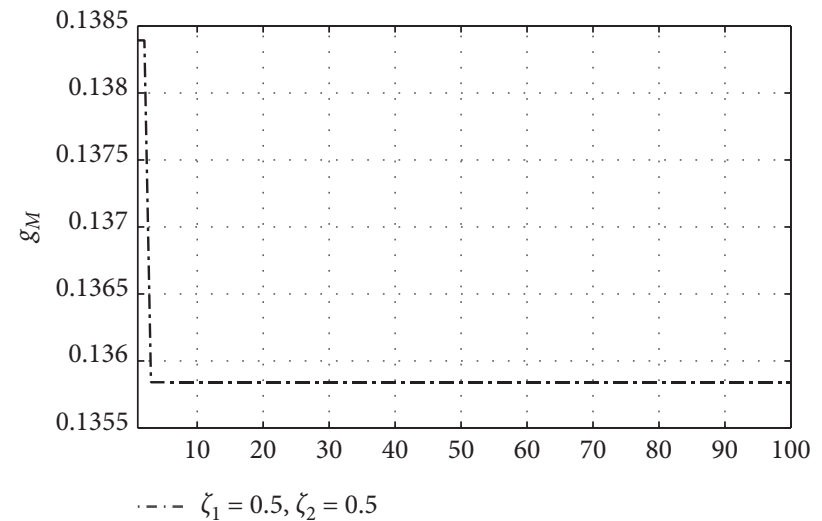

(b)

FIGURE 12: Continued. 


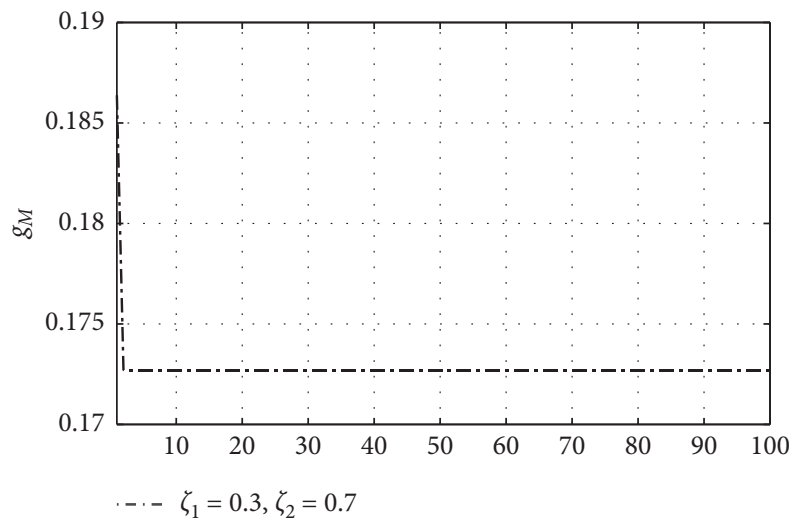

(c)

FIGURE 12: The relationship between iteration and objective function with different weight values.

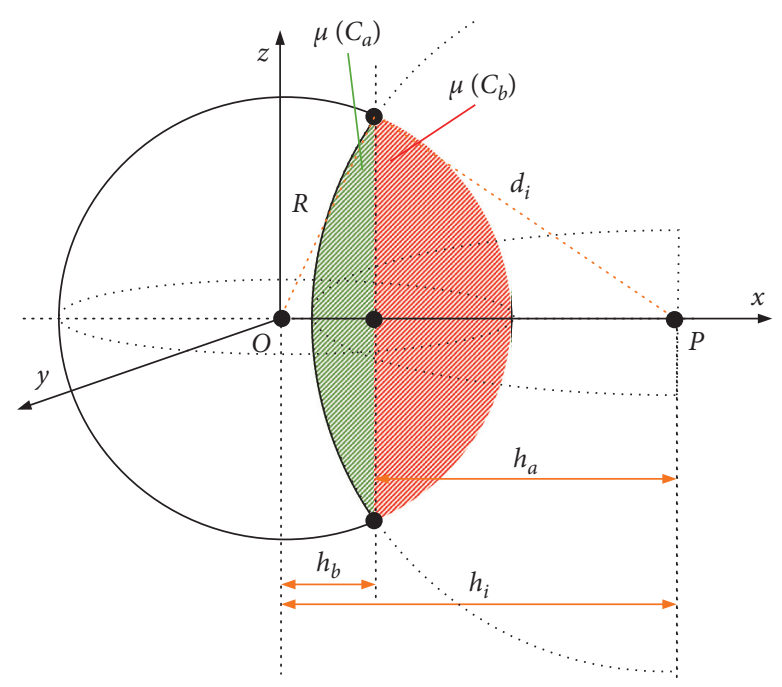

(a)

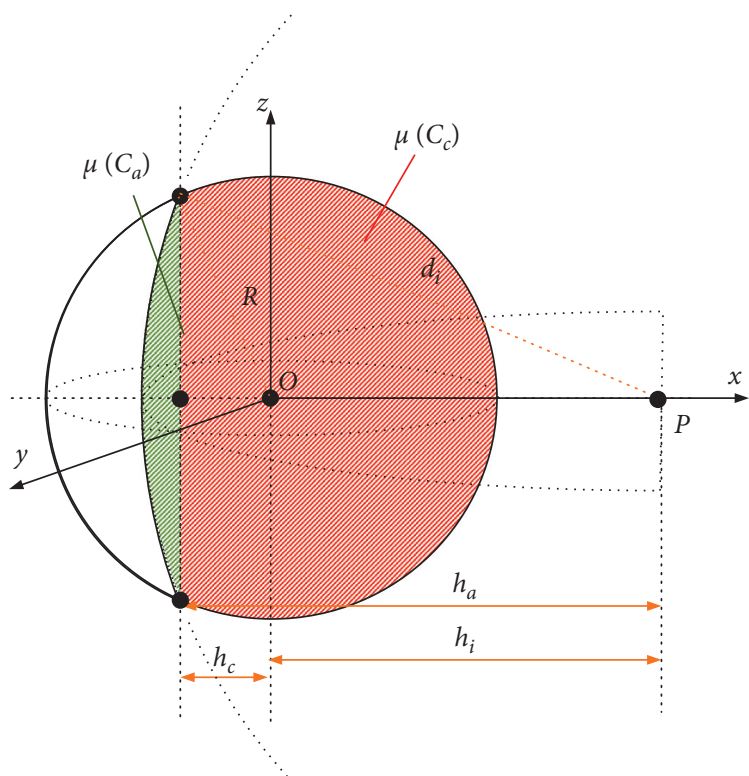

(b)

FIGURE 13: The transmitter-target distance distribution in SCFNR. (a) $d_{i} \in\left[h_{i}-R, h_{i}\right)$. (b) $d_{i} \in\left[h_{i}, h_{i}+R\right]$.

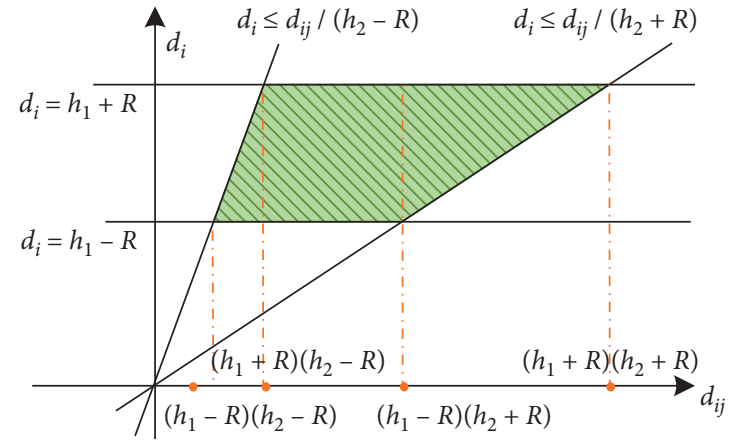

Figure 14: The feasible region with respect to $d_{i}$ and $d_{i j}$. 


\section{Conclusions}

In this paper, we study the optimization problem of the transmitter-receiver pairing of SCFNR for area coverage and target detection. Firstly, the novel concept of SCFNR integrated cluster flight spacecraft with netted radar is proposed. By establishing the mobility model for bistatic radar pair with twin-satellite mode, we have derived the radartarget distance distribution function and radar-target distance product distribution function with geometric probability method. Secondly, the radar-target distance distribution function and radar-target distance product distribution function with geometric probability method are proposed; we present the 0-1 grid coverage matrix for the bistatic radar and the transmitter-receiver paring matrix for SCFNR using the radar equation and the radar-target distance distribution function. Next, we describe the optimal problem of transmitter-receiver pairing of SCFNR for area coverage and target detection by defining $K$-grid coverage matrix. Finally, we propose new PSO-HA for the problem. We validate the effectiveness and reasonability of the proposed algorithm through numerical analysis. The numerical results can also be concluded as follows:

(1) Geometric topology of SCFNR with characteristic great dynamic and random leads to optimal paring scheme with characteristic time varying and random;

(2) When the paring matrix is optimal, the coverage level and detection probability with different weigh values had a slight change;
(3) SNR threshold had a great discernible impact on coverage and detection. In other words, the smaller the SNR threshold, the more achievable its coverage and detection will be.

In the future, to further develop the theory and application of SCFNR, we will study problems on target detection, localization, and tracking.

\section{Appendix}

Proof of Theorem 1. For distance distribution $F_{D_{i}}\left(d_{i}\right)$, we can obtain using equation (3), where $\mu(\Omega)=4 \pi R^{3} / 3$ and intersection volume $\mu\left(C_{0}\right)$ is unknown. In order to calculate $\mu\left(C_{0}\right)$, we categorize as follows:

(1) When $d_{i} \in\left[h_{i}-R, h_{i}\right)$, as shown in Figure 13(a), there exists $h_{a}+h_{b}=h_{i}, d_{i}^{2}-h_{a}^{2}=R^{2}-h_{b}^{2}$, thus $h_{a}=\left(h_{i}^{2}-R^{2}+d_{i}^{2} / 2 h_{i}\right), h_{b}=\left(h_{i}^{2}+R^{2}-d_{i}^{2} / 2 h_{i}\right)$, and the intersection volume $\mu\left(C_{0}\right)=\mu\left(C_{a}\right)+\mu\left(C_{b}\right)$. Actually, in Figure 13(a), $\mu\left(C_{a}\right)$ and $\mu\left(C_{b}\right)$ are one part of sphere $P$ with $d_{i}$ radius and sphere $O$ with $R$ radius, respectively. The methods to calculate $\mu\left(C_{a}\right)$ and $\mu\left(C_{b}\right)$ are the same.

For $\mu\left(C_{a}\right)$, let $x^{2}+y^{2}+z^{2}=d_{i}^{2}$ (see Figure 13(a) again); using triple integral, we can calculate it as follows:

$$
\mu\left(C_{a}\right)=\int_{h_{a}}^{d_{i}} \mathrm{~d} x \iint \mathrm{d} y \mathrm{~d} z=\int_{h_{a}}^{d_{i}} \pi\left(d_{i}^{2}-x^{2}\right) \mathrm{d} x=\frac{2 \pi d_{i}^{3}}{3}+\frac{\pi h_{a}^{3}}{3}-\pi d_{1}^{2} h_{a}
$$

In the same way, analogous, we can get $\mu\left(C_{b}\right)$, that is,

$$
\mu\left(C_{b}\right)=\frac{2 \pi R^{3}}{3}+\frac{\pi h_{b}^{3}}{3}-\pi R^{2} h_{b} .
$$

Hence, we have

$$
\mu\left(C_{0}\right)=\frac{2 \pi d_{i}^{3}}{3}+\frac{\pi h_{a}^{3}}{3}-\pi d_{i}^{2} h_{a}+\frac{2 \pi R^{3}}{3}+\frac{\pi h_{b}^{3}}{3}-\pi R^{2} h_{b} .
$$

Then, substituting equation (A.3) and $\mu(\Omega)=4 \pi R^{3} / 3$ into equation (3), $F_{D_{i}}\left(d_{i}\right)$ can be calculated as

$$
F_{D_{i}}\left(d_{i}\right)=\frac{2 d_{i}^{3}+h_{a}^{3}-3 h_{a} d_{i}^{2}+2 R^{3}+h_{b}^{3}-3 h_{b} R^{2}}{4 R^{3}} .
$$

(2) When $d_{i} \in\left[h_{i}, h_{i}+R\right]$, as shown in Figure 13(b), there exists $h_{a}-h_{c}=h_{i}, d_{i}^{2}-h_{a}^{2}=R^{2}-h_{c}^{2}$, thus
$h_{a}=\left(h_{i}^{2}-R^{2}+d_{i}^{2} / 2 h_{i}\right), \quad h_{c}=\left(-h_{i}^{2}-R^{2}+d_{i}^{2} / 2 h_{i}\right)$. The intersection volume $\mu\left(C_{0}\right)=\mu\left(C_{a}\right)+\mu\left(C_{c}\right)$. In the same way described in equation (A.1), we can calculate $\mu\left(C_{c}\right)$ as follows:

$$
\mu\left(C_{c}\right)=\frac{2 \pi R^{3}}{3}-\frac{\pi h_{c}^{3}}{3}+\pi R^{2} h_{c} .
$$

Hence, we have

$$
\mu\left(C_{0}\right)=\frac{2 \pi d_{i}^{3}}{3}+\frac{\pi h_{a}^{3}}{3}-\pi d_{i}^{2} h_{a}+\frac{2 \pi R^{3}}{3}-\frac{\pi h_{c}^{3}}{3}+\pi R^{2} h_{c} .
$$

Then, substituting equation (A.6) and $\mu(\Omega)=4 \pi R^{3} / 3$ into equation (3), $F_{D_{i}}\left(d_{i}\right)$ can be calculated as

$$
F_{D_{i}}\left(d_{i}\right)=\frac{2 d_{i}^{3}+h_{a}^{3}-3 h_{a} d_{i}^{2}+2 R^{3}-h_{c}^{3}+3 h_{c} R^{2}}{4 R^{3}} .
$$

To sum up, $F_{D_{i}}\left(d_{i}\right)$ is given by 


$$
F_{D_{i}}\left(d_{i}\right)= \begin{cases}\frac{2,}{2 d_{i}^{3}+h_{a}^{3}-3 h_{a} d_{i}^{2}+2 R^{3}+h_{b}^{3}-3 h_{b} R^{2}} & d_{i}<h_{i}-R, \\ 4 R^{3} & h_{i}-R \leq d_{i}<h_{i}, \\ \frac{2 d_{i}^{3}+h_{a}^{3}-3 h_{a} d_{i}^{2}+2 R^{3}-h_{c}^{3}+3 h_{c} R^{2}}{4 R^{3}}, & h_{i} \leq d_{i} \leq h_{i}+R, \\ 1, & h_{i}+R<d_{i} . \\ f_{D_{i j}}\left(d_{i j}\right)=\int_{-\infty}^{\infty} \frac{1}{\left|d_{i}\right|} f_{D_{i}}\left(d_{i}\right) f_{D_{j}}\left(\frac{d_{i j}}{d_{i}}\right) \mathrm{d} d_{i} .\end{cases}
$$

Proof of Theorem 2. Let $\left\|P S_{j R}\right\|=d_{j}$ be the distance between target $P$ and receiver, then $\left\|S_{i T} P\right\|\left\|P S_{j R}\right\|=d_{i} d_{j}=d_{i j}$ is the product of transmitter-target and target-receiver distances. To calculate the distribution function of $d_{i j}$, the accurate probability density functions of $d_{i}$ and $d_{j}$ are indispensable.

In Theorem 1, we have got the distance function $F_{D_{i}}\left(d_{i}\right)$, so the probability density function of $d_{i}$ is

$$
f_{D_{i}}\left(d_{i}\right)=\frac{\partial F_{D_{i}}\left(d_{i}\right)}{\partial d_{i}} .
$$

In the same way, we can also get $f_{D_{i}}\left(d_{j}\right)$. Since the two random variables $d_{i}$ and $d_{j}$ are independent, the probability density function of $d_{i j}$ can be obtained by
For convenience, let $h_{1}=\max \left\{h_{i}, h_{j}\right\} \quad$ and $h_{2}=\min \left\{h_{i}, h_{j}\right\}$. Obviously, $d_{i}>0$; thus, $f_{D_{i j}}\left(d_{i j}\right)$ can be rewritten as follows:

$$
f_{D_{i j}}\left(d_{i j}\right)=\int_{-\infty}^{+\infty} \frac{1}{d_{i}} f_{D_{i}}\left(d_{i}\right) f_{D_{j}}\left(\frac{d_{i j}}{d_{i}}\right) \mathrm{d} d_{i},
$$

where

$$
\begin{aligned}
& f_{D_{i}}\left(d_{i}\right)= \begin{cases}\frac{3\left(R^{2}-h_{1}^{2}\right) d_{i}+6 h_{1} d_{i}^{2}-3 d_{i}^{3}}{4 R^{3} h_{1}}, & h_{1}-R \leq d_{i} \leq h_{1}+R, \\
0, & \text { otherwise, }\end{cases} \\
& f_{D_{j}}\left(d_{j}\right)= \begin{cases}\frac{3\left(R^{2}-h_{2}^{2}\right) d_{j}+6 h_{2} d_{j}^{2}-3 d_{j}^{3}}{4 R^{3} h_{2}}, & h_{2}-R \leq d_{j} \leq h_{2}+R, \\
0, & \text { otherwise. }\end{cases}
\end{aligned}
$$

Thus, the distribution function of $d_{i j}$ is

$$
\begin{aligned}
F_{D_{i j}}\left(d_{i j}\right) & =\int_{\left(h_{1}-R\right)\left(h_{2}-R\right)}^{d_{i j}} f_{D_{i j}}\left(d_{i j}\right) \mathrm{d} d_{i j} \\
& =\int_{\left(h_{1}-R\right)\left(h_{2}-R\right)}^{d_{i j}} \int_{-\infty}^{+\infty} \frac{1}{d_{i}} f_{D_{i}}\left(d_{i}\right) f_{D_{j}}\left(\frac{d_{i j}}{d_{i}}\right) \mathrm{d} d_{i} \mathrm{~d} d_{i j} .
\end{aligned}
$$

When equation (A.13) is not equal to 0 , the feasible region with respect to $d_{i}$ and $d_{i j}$ is shown in Figure 14 . Therefore, for equation (A.13), we categorize as follows:

(1) When $\left(h_{1}-R\right)\left(h_{2}-R\right) \leq d_{i j}<\left(h_{1}+R\right)\left(h_{2}-R\right)$, we get 


$$
\begin{aligned}
F_{D_{i j}}^{1}\left(d_{i j}\right)= & \int_{\left(h_{1}-R\right)\left(h_{2}-R\right)}^{d_{i j}} \int_{\left(h_{1}-R\right)}^{d_{i j} /\left(h_{2}-R\right)} \frac{1}{d_{i}} f_{D_{i}}\left(d_{i}\right) f_{D_{j}}\left(\frac{d_{i j}}{d_{i}}\right) \mathrm{d} d_{i} \mathrm{~d} d_{i j} \\
= & \int_{\left(h_{1}-R\right)\left(h_{2}-R\right)}^{d_{i j}}\left(C_{1}^{1} d_{i j}+C_{2}^{1} d_{i j}^{2}+C_{3}^{1} d_{i j}^{3}\right) \mathrm{d} d_{i j}+\int_{\left(h_{1}-R\right)\left(h_{2}-R\right)}^{d_{i j}}\left(C_{4}^{1} d_{i j} \ln \frac{d_{i j}}{\left(h_{1}-R\right)\left(h_{2}-R\right)}+C_{5}^{1} d_{i j}^{2} \ln \frac{d_{i j}}{\left(h_{1}-R\right)\left(h_{2}-R\right)}\right) \mathrm{d} d_{i j} \\
& +\int_{\left(h_{1}-R\right)\left(h_{2}-R\right)}^{d_{i j}} C_{6}^{1} d_{i j}^{3} \ln \frac{d_{i j}}{\left(h_{1}-R\right)\left(h_{2}-R\right)} \mathrm{d} d_{i j} \\
= & \frac{C_{1}^{1} d_{i j}^{2}}{2}+\frac{C_{2}^{1} d_{i j}^{3}}{3}+\frac{C_{3}^{1} d_{i j}^{4}}{4}+\frac{C_{4}^{1} d_{i j}^{2}}{4}\left(2 \ln \frac{d_{i j}}{\left(h_{1}-R\right)\left(h_{2}-R\right)}-1\right) \\
& +\frac{C_{5}^{1} d_{i j}^{3}}{9}\left(3 \ln \frac{d_{i j}}{\left(h_{1}-R\right)\left(h_{2}-R\right)}-1\right) \\
& +\frac{C_{6}^{1} d_{i j}^{4}}{16}\left(4 \ln \frac{d_{i j}}{\left(h_{1}-R\right)\left(h_{2}-R\right)}-1\right)+C_{0}^{1}
\end{aligned}
$$

where

$$
\begin{aligned}
& C_{1}^{1}=\frac{9\left(h_{1}-R\right)\left(h_{2}-R\right)\left(3 h_{1} h_{2}+2 h_{1} R+2 h_{2} R+R^{2}\right)}{16 h_{1} h_{2} R^{6}}, \\
& C_{2}^{1}=-\frac{9\left(h_{1}+h_{2}\right)}{4 h_{1} h_{2} R^{5}}, \\
& C_{3}^{1}=\frac{1}{16 h_{1} h_{2} R^{6}}\left(\frac{-27 h_{1}+9 R}{2 h_{1}-2 R}+\frac{-27 h_{2}+9 R}{2 h_{2}-2 R}\right), \\
& C_{4}^{1}=\frac{9\left(h_{1}^{2}-R^{2}\right)\left(h_{2}^{2}-R^{2}\right)}{16 h_{1} h_{2} R^{6}}, \\
& C_{5}^{1}=\frac{9}{4 R^{6}}, \\
& C_{6}^{1}=\frac{9}{16 h_{1} h_{2} R^{6}}, \\
& C_{0}^{1}=-\frac{\left(h_{1}-R\right)^{3}\left(h_{2}-R\right)^{3}\left(h_{1}+3 R\right)\left(h_{2}+3 R\right)}{256 h_{1} h_{2} R^{6}} .
\end{aligned}
$$

(2) When $\left(h_{1}+R\right)\left(h_{2}-R\right) \leq d_{i j}<\left(h_{1}-R\right)\left(h_{2}+R\right)$, we get

$$
\begin{aligned}
F_{D_{i j}}^{2}\left(d_{i j}\right) & =\int_{\left(h_{1}+R\right)\left(h_{2}-R\right)}^{d_{i j}} \int_{\left(h_{1}-R\right)}^{\left(h_{1}+R\right)} \frac{1}{d_{i}} f_{D_{i}}\left(d_{i}\right) f_{D_{j}}\left(\frac{d_{i j}}{d_{i}}\right) \mathrm{d} d_{i} \mathrm{~d} d_{i j} \\
& =\int_{\left(h_{1}+R\right)\left(h_{2}-R\right)}^{d_{i j}}\left(C_{1}^{2} d_{i j}+C_{2}^{2} d_{i j}^{2}+C_{3}^{2} d_{i j}^{3}\right) \mathrm{d} d_{i j} \\
& =\frac{C_{1}^{2} d_{i j}^{2}}{2}+\frac{C_{2}^{2} d_{i j}^{3}}{3}+\frac{C_{3}^{2} d_{i j}^{4}}{4}+C_{0}^{2},
\end{aligned}
$$

where

$$
\begin{aligned}
C_{1}^{2}= & \frac{9}{16 h_{1} h_{2} R^{6}}\left(\left(h_{i}^{2}-R^{2}\right)\left(h_{2}^{2}-R^{2}\right) \ln \frac{\left(h_{1}+R\right)}{\left(h_{1}-R\right)}\right. \\
& \left.-2 R h_{1}\left(h_{2}^{2}-R^{2}\right)\right), \\
C_{2}^{2}= & \frac{9}{4 R^{6}} \ln \frac{\left(h_{1}+R\right)}{\left(h_{2}-R\right)}-\frac{18}{4 h_{1} R^{5}}, \\
C_{3}^{2}= & \frac{1}{16 h_{1} h_{2} R^{6}}\left(\frac{9 R-27 h_{1}}{2 h_{1}-2 R}+\frac{9 R+27 h_{2}}{2 h_{2}+2 R}+9 \ln \frac{\left(h_{1}+R\right)}{\left(h_{1}-R\right)}\right), \\
C_{0}^{2}= & F_{D_{i j}}^{1}\left(\left(h_{1}+R\right)\left(h_{2}-R\right)\right) \\
& -\frac{C_{1}^{2}\left(h_{1}+R\right)^{2}\left(h_{2}-R\right)^{2}}{2}-\frac{C_{2}^{2}\left(h_{1}+R\right)^{3}\left(h_{2}-R\right)^{3}}{3} \\
& -\frac{C_{3}^{2}\left(h_{1}+R\right)^{4}\left(h_{2}-R\right)^{4}}{4} .
\end{aligned}
$$


(3) When $\left(h_{1}-R\right)\left(h_{2}+R\right) \leq d_{i j}<\left(h_{1}+R\right)\left(h_{2}+R\right)$, we get

$$
\begin{aligned}
& F_{D_{i j}}^{3}\left(d_{i j}\right)= \int_{\left(h_{1}-R\right)\left(h_{2}+R\right)}^{d_{i j}} \int_{d_{i j} /\left(h_{2}+R\right)}^{\left(h_{1}-R\right)} \frac{1}{d_{i}} f_{D_{i}}\left(d_{i}\right) f_{D_{j}}\left(\frac{d_{i j}}{d_{i}}\right) \mathrm{d} d_{i} \mathrm{~d} d_{i j} \\
&= \int_{\left(h_{1}-R\right)\left(h_{2}+R\right)}^{d_{i j}}\left(C_{1}^{3} d_{i j}+C_{2}^{3} d_{i j}^{2}+C_{3}^{3} d_{i j}^{3}\right) \mathrm{d} d_{i j}+ \\
& \int_{\left(h_{1}-R\right)\left(h_{2}+R\right)}^{d_{i j}}\left(C_{4}^{3} d_{i j} \ln \frac{d_{i j}}{\left(h_{1}+R\right)\left(h_{2}+R\right)}+C_{5}^{3} d_{i j}^{2} \ln \frac{d_{i j}}{\left(h_{1}+R\right)\left(h_{2}+R\right)}\right) \mathrm{d} d_{i j} \\
&+\int_{\left(h_{1}-R\right)\left(h_{2}+R\right)}^{d_{i j}} C_{6}^{3} d_{i j}^{3} \ln \frac{d_{i j}}{\left(h_{1}+R\right)\left(h_{2}+R\right)} \mathrm{d} d_{i j} \\
&= \frac{C_{1}^{3} d_{i j}^{2}}{2}+\frac{C_{2}^{3} d_{i j}^{3}}{3}+\frac{C_{3}^{3} d_{i j}^{4}}{4}+\frac{C_{4}^{3} d_{i j}^{2}}{4}\left(2 \ln \frac{d_{i j}}{\left(h_{1}+R\right)\left(h_{2}+R\right)}-1\right)+\frac{C_{5}^{3} d_{i j}^{3}}{9}\left(3 \ln \frac{d_{i j}}{\left(h_{1}+R\right)\left(h_{2}+R\right)}-1\right) \\
&+\frac{C_{6}^{3} d_{3}^{4}}{16}\left(4 \ln \frac{d_{i j}}{\left(h_{1}+R\right)\left(h_{2}+R\right)}-1\right)+C_{0}^{3},
\end{aligned}
$$

where

$$
\begin{aligned}
& C_{1}^{3}=-\frac{9\left(h_{1}+R\right)\left(h_{2}+R\right)\left(3 h_{1} h_{2}-2 h_{1} R-2 h_{2} R+R^{2}\right)}{16 h_{1} h_{2} R^{6}}, \\
& C_{2}^{3}=-\frac{9\left(h_{1}+h_{2}\right)}{4 h_{1} h_{2} R^{5}} \\
& C_{3}^{3}=\frac{1}{16 h_{1} h_{2} R^{6}}\left(\frac{27 h_{1}+9 R}{2 h_{1}+2 R}+\frac{27 h_{2}+9 R}{2 h_{2}+2 R}\right) \text {, } \\
& C_{4}^{3}=-\frac{9\left(h_{1}^{2}-R^{2}\right)\left(h_{2}^{2}-R^{2}\right)}{16 h_{1} h_{2} R^{6}} \\
& C_{5}^{3}=-\frac{9}{4 R^{6}}, \\
& C_{6}^{3}=-\frac{9}{16 h_{1} h_{2} R^{6}} \\
& C_{0}^{3}=F_{D_{i j}}^{2}\left(\left(h_{1}-R\right)\left(h_{2}+R\right)\right)-\frac{C_{1}^{3}\left(h_{1}-R\right)^{2}\left(h_{2}+R\right)^{2}}{2}-\frac{C_{2}^{3}\left(h_{1}-R\right)^{3}\left(h_{2}+R\right)^{3}}{3} \\
& -\frac{C_{3}^{3}\left(h_{1}-R\right)^{4}\left(h_{2}+R\right)^{4}}{4}-\frac{C_{4}^{3} d_{i j}^{2}}{4}\left(2 \ln \frac{\left(h_{1}-R\right)}{\left(h_{1}+R\right)}-1\right)+\frac{C_{5}^{3} d_{i j}^{3}}{9}\left(3 \ln \frac{\left(h_{1}-R\right)}{\left(h_{1}+R\right)}-1\right)+\frac{C_{6}^{3} d_{i j}^{3}}{16}\left(3 \ln \frac{\left(h_{1}-R\right)}{\left(h_{1}+R\right)}-1\right)
\end{aligned}
$$




\section{Data Availability}

The data used to support the findings of this study are described in Section 6.1 of this article.

\section{Conflicts of Interest}

The authors declare that there are no conflicts of interest regarding the publication of this paper.

\section{Acknowledgments}

This research work was supported by the Guizhou Province Education Department Projects of China (KY[2017]031 and KY[2020]007).

\section{References}

[1] H.-Y. Zhao, Z.-J. Zhang, J. Liu, S. Zhou, J. Zheng, and W. Liu, "Target detection based on $F$-test in passive multistatic radar," Digital Signal Processing, vol. 79, pp. 1-8, 2018.

[2] Y. Zhao, Y. Zhao, and C. Zhao, "A novel algebraic solution for moving target localization in multi-transmitter multi-receiver passive radar," Signal Processing, vol. 143, pp. 303-310, 2018.

[3] X. Zhang, H. Li, and B. Himed, "Multistatic detection for passive radar with direct-path interference," IEEE Transactions on Aerospace and Electronic Systems, vol. 53, no. 2, pp. 915-925, 2017.

[4] A. R. Persico, P. Kirkland, C. Clemente, J. J. Soraghan, and M. Vasile, "CubeSat-based passive bistatic radar for space situational awareness: a feasibility study," IEEE Transactions on Aerospace and Electronic Systems, vol. 55, no. 1, pp. 476485, 2019.

[5] C. W. Wang, J. F. Pei, R. F. Wang, Y. L. Huang, and J. Y. Yang, "ExoMars spacecraft detection with European space surveillance bistatic radar," in Proceedings of the 2016 CIE International Conference on Radar (RADAR), pp. 10-13, Guangzhou, China, October 2016.

[6] L. Mazal and P. Gurfil, "Cluster flight algorithms for disaggregated satellites," Journal of Guidance, Control, and Dynamics, vol. 36, no. 1, pp. 124-135, 2013.

[7] A. Kandhalu and R. Rajkumar, "QoS-based resource allocation for next-generation spacecraft networks," in Proceedings of the IEEE 33rd Real Time Systems Symposium, pp. 163-172, San Juan, Puerto Rico, December 2012.

[8] S. Nag, C. K. Gatebe, and O. d. Weck, "Observing system simulations for small satellite formations estimating bidirectional reflectance," International Journal of Applied Earth Observation and Geoinformation, vol. 43, pp. 102-118, 2015.

[9] H. Zhang and P. Gurfil, "Distributed control for satellite cluster flight under different communication topologies," Journal of Guidance, Control, and Dynamics, vol. 39, pp. 617-627, 2015.

[10] T. Yan, S. Hu, and J. Mo, "Path formation time in the noiselimited fractionated spacecraft network with FDMA," International Journal of Aerospace Engineering, vol. 2018, Article ID 9124132, , 2018.

[11] J. Mo, S. Hu, T. Yan, X. Song, and Y. Shi, “Transmit power allocation with connectivity probability for Multi-QoS in cluster flight spacecraft network," Wireless Communications and Mobile Computing, vol. 2020, Article ID 8676835, , 2020.
[12] J. Mo, S. Hu, Y. Shi, X. Song, and T. Yan, "Nodal distance distributions in cluster flight spacecraft network," Mathematical Methods in the Applied Sciences, vol. 43, no. 17, 2020.

[13] S. Cakaj, B. Kamo, and A. Rakipi, "The coverage analysis for low earth orbiting satellites at low elevation," International Journal of Advanced Computer Science and Applications, vol. 5, no. 6, 2014.

[14] P. Zong and S. Kohani, "Optimal satellite LEO constellation design based on global coverage in one revisit time," International Journal of Aerospace Engineering, vol. 2019, Article ID 4373749, , 2019.

[15] W. Beide, "The nature of bistatic and multistatic radar," in Proceedings of the 2001 CIE International Conference on Radar, pp. 882-884, Edinburgh, UK, 2001.

[16] S. D. Blunt and K. Gerlach, "Aspects of multistatic adaptive pulse compression," in Proceedings of the International IEEE Radar Conference, pp. 104-108, Washington, DC, USA, June 2005.

[17] I. Bradaric, G. Capraro, D. D. Weiner, and M. C. Wicks, "Multistatic radar systems signal processing," in Proceedings of the IEEE Radar Conference, pp. 106-113, New Delhi, India, May 2006.

[18] D. Bruyère and N. A. Goodman, "Performance of multistatic space-time adaptive processing," in Proceedings of the IEEE Radar Conference, pp. 533-538, Verona, NY, USA, 2006.

[19] E. Hanle, "Survey of bistatic and multistatic radar," IEE Proceedings F Communications, Radar and Signal Processing, vol. 133, no. 7, pp. 587-595, 1986.

[20] M.-Z. Shieh, S.-C. Tsai, and M.-C. Yang, "On the inapproximability of maximum intersection problems," Information Processing Letters, vol. 112, no. 19, pp. 723-727, 2012.

[21] E. T. Bogue, C. C. Souza, E. C. Xavier, and A. S. Freire, "An integer programming formulation for the maximum $k$-subset intersection problem," Lecture Notes in Computer Science, vol. 8596, pp. 87-99, 2014.

[22] X. W. Gong, J. S. Zhang, C. Cochran, and K. Xing, "Optimal placement for barrier coverage in bistatic radar sensor networks," IEEE/ACM Transactions on Networking, vol. 24, no. 1, pp. 259-271, 2014.

[23] B. Wang, J. Y. Chen, W. Y. Liu, and L. T. Yang, "Minimum cost placement of bistatic radar sensors for belt barrier coverage," IEEE Transactions on Computers, vol. 65, no. 2, pp. 577-588, 2015.

[24] Q. Q. Yang, S. B. He, and J. M. Chen, "Energy-efficient area coverage in bistatic radar sensor networks," in Proceedings of the Global Communications Conference, pp. 280-285, Atlanta, GA, USA, December 2013.

[25] X. W. Gong, J. S. Zhang, and D. A. Cochran, "A coverage theory of bistatic radar networks: worst-case intrusion path and optimal deployment," Networking and Internet Architecture, http://arxiv.org/abs/1206.1355, 2012.

[26] R. Q. Wang, S. B. He, J. M. Chen, Z. G. Shi, and F. Hou, "Energy-efficient barrier coverage in bistatic radar sensor networks," in Proceedings of the IEEE International Conference on Communications (ICC), pp. 8-12, London, UK, 2015.

[27] J. He, Z. Xing, R. Hu et al., "Directional antenna intelligent coverage method based on traversal optimization algorithm," Computers, Materials \& Continua, vol. 60, no. 2, pp. 527-544, 2019.

[28] C. Duan, J. Feng, H. Chang, J. Pan, and L. Duan, "Research on sensor network coverage enhancement based on non-cooperative games," Computers, Materials \& Continua, vol. 60, no. 3, pp. 989-1002, 2019. 
[29] C. Chen and Y. Lin, "Development of a data-driven ANFIS model by using PSO-LSE method for nonlinear system identification," Intelligent Automation and Soft Computing, vol. 25, no. 2, pp. 319-327, 2019.

[30] W. Zhang, W. Shi, and B. Sun, "BDI agent and QPSO-based parameter optimization for a marine generator excitation controller," Intelligent Automation and Soft Computing, vol. 25, no. 3, pp. 423-431, 2019.

[31] J. Wang, Y. Gao, C. Zhou, R. Simon Sherratt, and L. Wang, "Optimal coverage multi-path scheduling scheme with multiple mobile sinks for WSNs," Computers, Materials \& Continua, vol. 62, no. 2, pp. 695-711, 2020.

[32] N. Pierdicca, L. De Titta, L. Pulvirenti, and G. Della Pietra, "Bistatic radar configuration for soil moisture retrieval: analysis of the spatial coverage," Sensors, vol. 9, no. 9, pp. 7250-7265, 2009.

[33] M. D. Errico and A. Moccia, "Remote sensing satellite formation for bistatic synthetic aperture radar observation," Sensors, Systems, and Next-Generation Satellites V, vol. 4540, 2001.

[34] R. R. Scherberger, H. Kaess, S. Brückner, and M. G. Gao, "Studies on the action of an anticholinergic agent in combination with a tranquilizer on gastric juice secretion in man," in Proceedings of the 2008 9th International Conference on Signal Processing, pp. 1460-1463, October 2008.

[35] J. H. Liu and G. H. Liao, "Spaceborne-airborne bistatic radar clutter modeling and analysis," in Proceedings of the 2011 IEEE CIE International Conference on Radar, pp. 24-27, Chengdu, China, October 2011.

[36] J. Huang, Y. Su, L. Huang, W. Liu, and F. Wang, "An optimized snapshot division strategy for satellite network in GNSS," IEEE Communications Letters, vol. 20, no. 12, pp. 2406-2409, 2016.

[37] N. J. Willis and H. D. Griffifiths, "Advances in bistatic radar (book review)," IEEE Aerospace and Electronic Systems Magazine, vol. 23, no. 7, p. 46, 2008.

[38] L. E. Miller, "Distribution of link distances in a wireless network," Journal of Research of the National Institute of Standards and Technology, vol. 106, no. 2, pp. 401-412, 2001.

[39] C. C. Tseng, H. T. Chen, and K. C. Chen, "On the distance distributions of the wireless ad hoc networks," in Proceedings of the 2006 IEEE 63rd Vehicular Technology Conference, pp. 772-776, Melbourne, Australia, 2006.

[40] D. Patel and M. J. Jha, "Hungarian method based resource scheduling algorithm in cloud computing," International Journal of Advance Research and Innovative Ideas in Education, vol. 2, pp. 54-59, 2016.

[41] H. Nakayama, "Multi-objective optimization and its engineering applications," Dagstuhl Seminar Proceedings, vol. 2005, Article ID 04461, 2005.

[42] I. Ibrahim, Z. M. Yusof, S. W. Nawawi et al., "A novel multistate particle swarm optimization for discrete combinatorial optimization problems," in Proceedings of the 2012 Fourth International Conference on Computational Intelligence, Modelling and Simulation, pp. 25-27, Kuantan, Malaysia, September 2012.

[43] M. Rosendo and A. Pozo, "Applying a discrete particle swarm optimization algorithm to combinatorial problems," in Proceedings of the 2010 Eleventh Brazilian Symposium on Neural Networks, pp. 23-28, Paulo, Brazil, October 2010.

[44] X. Yu, W.-N. Chen, T. Gu et al., "Set-based discrete particle swarm optimization based on decomposition for permutation-based multiobjective combinatorial optimization problems," IEEE Transactions on Cybernetics, vol. 48, no. 7, pp. 2139-2153, 2018.

[45] J. Kennedy and R. Eberhart, "Particle swarm optimization," in Proceedings of IEEE International Conference on Neural Networks, pp. 1942-1948, Perth, Australia, 1995.

[46] Y. Yang, W. Yi, T. Zhang et al., "Fast optimal antenna placement for distributed MIMO radar with surveillance performance," IEEE Signal Processing Letters, vol. 22, no. 11, pp. 1955-1959, 2015. 